\title{
A COMPARISON OF X-RAY AND MID-INFRARED SELECTION OF OBSCURED ACTIVE GALACTIC NUCLEI
}

\author{
Megan E. Eckart ${ }^{1,2,7}$, Ian D. McGreer ${ }^{3,4},{\text { Daniel } \text { Stern }^{5}, \text { Fiona A. Harrison }^{1} \text {, And David J. Helfand }}^{3,6}$ \\ ${ }^{1}$ Space Radiation Laboratory, Mail Stop 290-17, California Institute of Technology, Pasadena, CA 91125, USA \\ 2 NASA Goddard Space Flight Center, Code 662, Greenbelt, MD 20771, USA \\ ${ }^{3}$ Department of Astronomy, Columbia University, 550 West 120th Street, New York, NY 10027, USA \\ ${ }^{4}$ Steward Observatory, University of Arizona, 933 North Cherry Avenue, Tucson, AZ 85721, USA \\ ${ }^{5}$ Jet Propulsion Laboratory, California Institute of Technology, Mail Stop 169-506, Pasadena, CA 91109, USA \\ ${ }^{6}$ Quest University Canada, 3200 University Blvd., Squamish, BC V8B0N8, Canada \\ Received 2009 March 15; accepted 2009 November 12; published 2009 December 14
}

\begin{abstract}
We compare the relative merits of active galactic nuclei (AGNs) selection at X-ray and mid-infrared wavelengths using data from moderately deep fields observed by both Chandra and Spitzer. The X-ray-selected AGN sample and associated photometric and spectroscopic optical follow-up are drawn from a subset of fields studied as part of the Serendipitous Extragalactic X-ray Source Identification (SEXSI) program. Mid-infrared data in these fields are derived from targeted and archival Spitzer imaging, and mid-infrared AGN selection is accomplished primarily through application of the Infrared Array Camera (IRAC) color-color AGN "wedge" selection technique. Nearly all X-ray sources in these fields which exhibit clear spectroscopic signatures of AGN activity have mid-infrared colors consistent with IRAC AGN selection. These are predominantly the most luminous X-ray sources. X-ray sources that lack high-ionization and/or broad lines in their optical spectra are far less likely to be selected as AGNs by mid-infrared color selection techniques. The fraction of X-ray sources identified as AGNs in the mid-infrared increases monotonically as the X-ray luminosity increases. Conversely, only $22 \%$ of mid-infrared-selected AGNs are detected at X-ray energies in the moderately deep $\left(\left\langle t_{\exp }\right\rangle \approx 100 \mathrm{ks}\right)$ SEXSI Chandra data. We hypothesize that IRAC sources with AGN colors that lack X-ray detections are predominantly high-luminosity AGNs that are obscured and/or lie at high redshift. A stacking analysis of X-ray-undetected sources shows that objects in the mid-infrared AGN selection wedge have average X-ray fluxes in the 2-8 keV band 3 times higher than sources that fall outside the wedge. Their X-ray spectra are also harder. The hardness ratio of the wedge-selected stack is consistent with moderate intrinsic obscuration, but is not suggestive of a highly obscured, Compton-thick source population. It is evident from this comparative study that in order to create a complete, unbiased census of supermassive black hole growth and evolution, a combination of sensitive infrared, X-ray, and hard X-ray selection is required. We conclude by discussing what samples will be provided by upcoming survey missions such as WISE, eROSITA, and NUSTAR.
\end{abstract}

Key words: galaxies: active - infrared: galaxies - X-rays: galaxies

Online-only material: color figures, machine-readable table

\section{INTRODUCTION}

The tight correlation of nuclear black hole mass with the velocity dispersion and mass of the galactic bulge (e.g., Magorrian et al. 1998; Ferrarese \& Merritt 2000; Tremaine et al. 2002) implies that the growth and evolution of galaxies is closely linked to the growth and evolution of the supermassive black holes which reside in (at least) all massive galaxies (e.g., Kauffmann \& Haehnelt 2000; Heckman 2008). Indeed, recent theoretical work suggests that feedback from active galactic nuclei (AGNs) plays a dominant role in establishing the present-day appearances of galaxies, providing a natural, physical explanation for both cosmic downsizing and the possibly related bimodality in local galaxy properties (e.g., Scannapieco et al. 2005; Hopkins et al. 2008; Cattaneo et al. 2009). However, obtaining an unbiased census of black holes in the universe remains challenging, hampering our ability to fully probe this connection.

Most surveys for active galaxies are severely biased toward unobscured (type 1) AGNs since nuclear emission in such sources dominates over host galaxy light at most wavelengths, making type 1 AGNs both more readily identifiable and easier to

\footnotetext{
7 NASA Postdoctoral Program (NPP) Fellow.
}

follow up spectroscopically. However, both unified AGN models (e.g., Antonucci 1993; Urry \& Padovani 1995) and the shape of the X-ray background suggest a population of obscured (type 2) sources which outnumber the type 1 AGN by up to a factor of 10 (e.g., Comastri et al. 1995; Treister et al. 2004; Gilli et al. 2007). Determining the ratio of unobscured to obscured AGNs as a function of luminosity and redshift can directly constrain the growth history of supermassive black holes, and help to quantify their influence on the evolution of their host galaxies (e.g., Ueda et al. 2003; Barger et al. 2005; Hasinger et al. 2005; Hopkins et al. 2007). Since different search techniques for obscured AGNs suffer from different biases, the problem is best addressed through multiwavelength studies.

The hard (2-10 keV) X-ray and mid-infrared wavebands provide powerful, complementary methods for identifying and studying AGNs over a wide range of intrinsic obscuration. Radiation seen from active galaxies at X-ray energies is primarily due to direct emission from accretion processes near the central supermassive black hole, with higher energy photons less susceptible to absorption, while mid-infrared radiation (rest-frame $\lambda_{0} \gtrsim 2 \mu \mathrm{m}$ ) is typically dominated by emission from dusty obscuring material surrounding the AGN central 
engine, and is likewise relatively immune to absorption (e.g., Krolik 1999). Hard X-ray and mid-infrared surveys of AGN will therefore sample a wide range of intrinsic absorption and, in this regard, will be both less biased and more complete than surveys in the optical (e.g., Richards et al. 2006) and soft X-ray $(E \lesssim 2.4 \mathrm{keV}$; e.g., Hasinger et al. 1998; Schmidt et al. 1998) bands, both of which suffer from strong attenuation by even moderate $\left(N_{H} \gtrsim 10^{21} \mathrm{~cm}^{-2}\right)$ columns of dusty, obscuring material. However, AGN samples selected in the $2-10 \mathrm{keV}$ X-ray and infrared bands will also suffer incompleteness. Examples exist of AGNs identified from optical spectroscopy which remain undetected even in the deepest X-ray images yet obtained (e.g., Steidel et al. 2002); similarly, there are examples of optically selected AGNs not identified in infrared surveys (e.g., Stern et al. 2005). Conversely, X-ray missions have identified AGNs whose optical spectra are devoid of AGN signatures (e.g., $\mathrm{X}$-ray bright, optically normal galaxies, or "XBONGs;" Comastri et al. 2002). Therefore, a complete census will necessarily require combining selection techniques from several spectral regimes.

The current generation of X-ray telescopes has dramatically advanced our knowledge of the AGN population (see Brandt \& Hasinger 2005 for a review). The Chandra X-ray Observatory (Weisskopf et al. 1996) provides a large collecting area, a moderate field of view (FOV), and exquisite angular resolution $\left(<1^{\prime \prime}\right)$ from 0.5 to $8 \mathrm{keV}$. These capabilities have allowed Chandra extragalactic surveys to efficiently select and optically identify large samples of AGN in the 2-8 keV range. Observing in this hard X-ray energy band means that even sources shrouded by considerable obscuring column densities (up to $10^{24} \mathrm{~cm}^{-2}$ ) can be detected at low redshift, and sources with even higher column densities can be detected out to $z \gtrsim 2$. Previous sensitive $\mathrm{X}$-ray telescopes were primarily restricted to energies below $\sim 2 \mathrm{keV}$ and thus missed many of the obscured AGNs, a source population that has long been theorized to explain the mismatch in spectral shape between the $2-10 \mathrm{keV}$ X-ray background $(\Gamma \approx 1.4)$ and the unobscured active galaxies which dominate the source counts at soft $\mathrm{X}$-ray energies $(\Gamma \approx 1.9$; Nandra \& Pounds 1994).

The 2003 launch of the Spitzer Space Telescope (Werner et al. 2004) opened a new era in mid-infrared observations, providing orders of magnitude improvement in sensitivity in the 3.6-160 $\mu \mathrm{m}$ band. The increased sensitivity, combined with the large FOV, allows for the first time efficient long-wavelength survey capabilities. The primary imaging cameras on Spitzer are the Infrared Array Camera (IRAC; Fazio et al. 2004), providing simultaneous imaging at 3.6, 4.5, 5.8, and $8 \mu \mathrm{m}$, and the Multiband Imaging Photometer for Spitzer (MIPS; Rieke et al. 2004), providing simultaneous imaging at 24,70 , and $160 \mu \mathrm{m}$.

Several methods have been developed to select AGNs based on their Spitzer colors (Lacy et al. 2004; Stern et al. 2005; Alonso-Herrero et al. 2006). These methods exploit the difference in the typical spectral energy distribution (SED) of AGNs compared to "normal" galaxies. The near-infrared emission of both typical galaxies and vigorously star-forming galaxies is primarily produced by a thermal stellar population, resulting in SEDs peaked near the rest-frame $1.6 \mu \mathrm{m}$ "bump." This bump, which is caused by the minimum in the opacity of the $\mathrm{H}^{-}$ion near $1.6 \mu \mathrm{m}$ (e.g., John 1988), is a feature of almost all stellar populations (e.g., Wright et al. 1994; Simpson \& Eisenhardt 1999), whereas AGN-dominated SEDs have a non-thermal, roughly power-law shape (for $\lambda \lesssim 10 \mu \mathrm{m}$ ). At longer wave- lengths contributions from stellar blackbody emission is low, while radiation from the obscuring dust near the central engine provides strong, relatively isotropic emission which is quite distinct from stellar light (although the shape of the AGN SED does somewhat depend on viewing angle, e.g., Elitzur 2008). Stern et al. (2005), for example, suggested a selection technique exploiting the fact that, for AGN, the long-wavelength side of the $1.6 \mu \mathrm{m}$ stellar peak does not decline; the technique uses an empirically determined "wedge" in IRAC color-color space ([3.6]-[4.5] versus [5.8]-[8.0]) that preferentially contains AGNs as compared to normal galaxies or Galactic stars. Combining a sample of 10,000 $R<21.5$ spectroscopically identified sources from the AGN and Galaxy Evolution Survey (AGES; C. S. Kochanek et al. 2010, in preparation) and mid-infrared observations from the IRAC Shallow Survey (Eisenhardt et al. 2004), Stern et al. (2005) defined mid-infrared AGN selection criteria which robustly identify broad- and narrow-lined AGNs, with only $18 \%$ sample contamination from galaxies $(17 \%)$ and stars $(1 \%)$. The true sample contamination is likely lower, since many of the spectroscopically normal galaxies may harbor active nuclei (i.e., are mid-infrared versions of XBONGs). Working from the full spectroscopically defined AGES sample, Stern et al. (2005) found that the wedge selects $91 \%$ of the broad-lined AGNs, $40 \%$ of the narrow-lined AGNs, and fewer than 3\% of the normal galaxies.

This paper presents an exploration of the relative strengths of Chandra and Spitzer as black-hole finders, using a subset of six fields from the Serendipitous Extragalactic X-ray Source Identification program (SEXSI; Harrison et al. 2003; Eckart et al. 2005, 2006) for which we have obtained mid-infrared coverage with Spitzer. In addition to examining the properties of $\sim 250$ hard X-ray-selected AGNs, we extend our sensitivity to X-ray emission using a stacking analysis on sources with different mid-infrared characteristics. This enables us to make a comprehensive comparison of the relative characteristics of AGN samples selected from their X-ray and mid-infrared properties.

We organize the paper as follows: Section 2 introduces the SEXSI program and presents the complementary mid-infrared observations and the resulting source catalog; Section 3 discusses the mid-infrared properties of the $2-10 \mathrm{keV}$ SEXSI sources; Section 4 presents the mean X-ray properties of $\mathrm{X}$-ray-non-detected Spitzer sources; Section 4.3 provides a discussion; and Section 5 summarizes our conclusions. Luminosity calculations assume a standard cosmology used in our previous work (Eckart et al. 2006), $\Omega_{0}=0.3, \Lambda=0.7$, and $H_{0}=65 \mathrm{~km} \mathrm{~s}^{-1} \mathrm{Mpc}^{-1}$.

\section{OBSERVATIONS, DATA REDUCTION, AND SOURCE CATALOG}

We have expanded the multiwavelength data available for six SEXSI fields by obtaining Spitzer imaging observations. All six fields have deep Chandra X-ray images, optical imaging, and extensive, deep optical spectroscopy-all of which has been published in Harrison et al. (2003), Eckart et al. (2005), and Eckart et al. (2006). In this paper, we present Spitzer archival and/or targeted observations from 3.6 to $24 \mu \mathrm{m}$ from these fields. Below we briefly review the SEXSI program, describe the complementary Spitzer sample and data reduction, and present the IRAC and MIPS photometry and source catalog for all 2$10 \mathrm{keV}$ SEXSI X-ray sources. 
Table 1

Spitzer Observations of SEXSI Fields

\begin{tabular}{lccccc}
\hline \hline Cluster Field & $z$ & $\begin{array}{c}\text { Chandra } \\
\text { Exp. Time }\end{array}$ & $\begin{array}{c}\text { Spitzer } \\
\text { Instrument }\end{array}$ & PID $^{\mathrm{b}}$ & Notes \\
\hline CL0848+44 & 1.27 & $186 \mathrm{ks}$ & IRAC & 00017 & \\
& & & IRAC & 00064 & \\
& & & IRAC & 20694 & Wider FOV \\
& & & MIPS & 00083 & \\
RXJ0910+54 & 1.11 & \multirow{2}{*}{$171 \mathrm{ks}$} & MIPS & 20694 & Wider FOV \\
& & & IRAC & 00017 & \\
& & & IRAC & 20808 & Wider FOV \\
& & & MIPS & 00083 & \\
RXJ1317+29 & 0.81 & $111 \mathrm{ks}$ & IRAC & 00017 & Wider FOV \\
BD1338+29 & 0.64 & \multirow{2}{*}{$38 \mathrm{ks}$} & IRAC & 20808 & \\
& & & MIPS & 20808 & \\
RXJ1716+67 & 0.81 & $52 \mathrm{ks}$ & IRAC & 00017 & \\
& & & IRAC & 20808 & Wider FOV \\
& & & MIPS & 20808 & \\
RXJ2247+03 & 0.18 & \multirow{2}{*}{$49 \mathrm{ks}$} & IRAC & 20808 & \\
& & & MIPS & 20808 & \\
& & & & &
\end{tabular}

Notes.

a This column provides the Chandra exposure times after removal of background flares.

b Spitzer program identifier (PID) 00017: distant X-ray galaxy clusters (PI: Fazio; GTO); PID 00064, combined program (PI: Fazio; GTO); PID 00083, use of massive clusters as cosmological lenses (PI: Rieke; GTO); PID 20694, IRAC and MIPS mapping of galaxy populations in a supercluster at $z=1.27$ (PI: Stanford; GO2); PID 20808, SEXSI (PI: Stern; GO2).

\subsection{SEXSI X-ray Sample and Optical Followup}

The SEXSI survey was designed to obtain a significant sample of spectroscopically identified objects in the $2-10 \mathrm{keV}$ flux range from a few times $10^{-13}$ to $10^{-15} \mathrm{erg} \mathrm{cm}^{-2} \mathrm{~s}^{-1}$, to provide a medium-area, medium-depth sample to complement both the wide-area, shallow surveys (e.g., HELLAS, La Franca et al. 2002; ASCA Large Sky Survey, Akiyama et al. 2000; ASCA Medium Sensitivity Survey, Akiyama et al. 2003) and deep, pencil-beam surveys (e.g., CDF-N, Alexander et al. 2003; Barger et al. 2003; CDF-S, Rosati et al. 2002; Szokoly et al. 2004). To accomplish this, SEXSI covers more than $2 \mathrm{deg}^{2}$ of sky, comprised of 27 archival Chandra ACIS images. The resulting source sample consists of 1034 hard X-ray-selected sources (Harrison et al. 2003) with complete $R$-band imaging (Eckart et al. 2005) and optical spectroscopy for $477(46 \%)$ of the sources (Eckart et al. 2006). The vast majority of these sources are active galaxies with $L_{x}(2-10 \mathrm{keV}) \gtrsim 10^{43} \mathrm{erg} \mathrm{s}^{-1}$. Just over half of these AGNs have optical spectra that show typical AGN lines which we classify as either broad-lined AGNs (BLAGNs) or narrow-lined AGNs (NLAGNs). Extragalactic sources which exhibit optical spectra devoid of the typical highionization AGN lines are called emission-line galaxies (ELGs) or absorption-line galaxies (ALGs); such sources are often referred to as XBONGs in the literature. See Eckart et al. (2006) for more details on the SEXSI spectroscopic classifications.

\subsection{Spitzer Mid-infrared Imaging}

We obtained mid-infrared imaging for six SEXSI fields through both archival and targeted Spitzer programs (see Table 1). These programs include imaging at 3.6, 4.5, 5.8, and $8 \mu \mathrm{m}$ from IRAC and imaging at $24 \mu \mathrm{m}$ from MIPS. Fields were chosen both to optimize use of archival Spitzer data and to target SEXSI fields with the most complete spectroscopic coverage.
Program ID 20808 was designed specifically for the SEXSI follow-up, with typical exposure times of $600 \mathrm{~s}$ for IRAC observations and $1000 \mathrm{~s}$ for MIPS observations. Because the SEXSI data were drawn from the Chandra public archive, the fields are not "blank": the target sources in the six SEXSI Spitzer fields are all $z>0.18$ galaxy clusters, five of which have $0.64<z<1.3$ (see Table 1).

\subsubsection{IRAC Observations and Reductions}

Our IRAC data reduction begins with the basic calibrated data (BCD) output from the Spitzer Science Center pipeline (ver. 10.0.1). Note that Spitzer BCD images have surface brightness units in $\mathrm{MJy} \mathrm{sr}^{-1}$, where $1 \mathrm{MJy} \mathrm{sr}^{-1}$ is equal to $23.5 \mu \mathrm{Jy} \operatorname{arcsec}^{-2}$. Most of the IRAC observations consist of six dithered $100 \mathrm{~s}$ exposures. For each IRAC channel, the BCDs were mosaicked using the MOPEX package (Makovoz $\&$ Khan 2005) and resampled by a factor of 2 to give a pixel scale of 0.'61. The MOPEX outlier (e.g., cosmic ray, bad pixel) rejection was optimized for the regions of deepest coverage in the centers of the images. Source extraction was performed with SExtractor (Bertin \& Arnouts 1996) both in single-image mode to produce independent source catalogs in all four IRAC bands and in dual-image mode using the $3.6 \mu \mathrm{m}$ channel for source detection. All IRAC BCD images have astrometry derived from the Two Micron All Sky Survey (2MASS) and are good to better than 0.5 .

In general, we use a $5^{\prime \prime}$ diameter aperture for IRAC photometry. For the small fraction of the sources $(\sim 5 \%)$ that are spatially resolved and larger than this, we use a $7^{\prime \prime}$ diameter aperture. We select the apertures to ensure a fixed extraction area similar to the isophotal area; this same methodology was adopted by both the Spitzer First Look Survey (FLS; Lacy et al. 2005) and the Spitzer High-Redshift Radio Galaxy program (SHzRG; Seymour et al. 2007). The aperture corrections, applied after the conversion from surface brightness to flux density, are 1.167, $1.213,1.237$, and 1.466 in the four IRAC bands, respectively, for the $5^{\prime \prime}$ apertures. The corresponding corrections for the $7^{\prime \prime}$ apertures are smaller $(1.100,1.130,1.109$, and 1.198, respectively). We derive total uncertainties on the resulting IRAC flux densities by adding in quadrature the statistical uncertainties (derived from SExtractor) to a conservative $10 \%$ estimate of the systematic uncertainties. The latter includes several effects (e.g., Hora et al. 2008): uncertainties in the IRAC zero point, field-dependent color corrections, pixel phase effects (e.g., zero point corrections that depend on the position of a source relative to the nearest pixel center), and variation of the pixel scale across the FOV. For nondetections we calculate $5 \sigma$ upper limits. A small number of sources are recovered by SExtractor with signal-to-noise ratios below $5(3<\mathrm{S} / \mathrm{N}<5)$; examination of the images shows that these are valid detections, generally near the edge of the mosaic. We flag these sources in the catalog and exclude them from the analysis presented here.

\subsubsection{MIPS Observations and Reductions}

MIPS observations obtained for this program (e.g., PID 20808; see Table 1) consist of two cycles of 30 s exposures with a $3 \times 3$ dither pattern at $24 \mu \mathrm{m}$. This observing strategy provides an average (median) exposure time of $1290 \mathrm{~s}(877 \mathrm{~s})$ across our combined fields of view. To process the MIPS data, the BCDs provided by the Spitzer Science Center were first median filtered to remove a stripe pattern artifact in the individual frames. We then mosaicked the data with MOPEX using standard inputs to produce final, stacked images with the native 
Table 2

SEXSI-Spitzer Catalog

\begin{tabular}{|c|c|c|c|c|c|c|c|c|c|c|c|c|c|c|}
\hline $\begin{array}{c}\text { CXOSEXSI } \\
\text { (1) } \\
\end{array}$ & $\begin{array}{l}\Delta \alpha \\
\left({ }^{\prime \prime}\right) \\
(2) \\
\end{array}$ & $\begin{array}{l}\Delta \delta \\
\left({ }^{\prime \prime}\right) \\
(3) \\
\end{array}$ & $\begin{array}{c}S_{2-10} \\
\left(10^{-15}\right) \\
(4) \\
\end{array}$ & $\begin{array}{l}\text { HR } \\
\text { (5) } \\
\end{array}$ & $\begin{array}{c}R \\
(\mathrm{mag}) \\
(6)\end{array}$ & $\begin{array}{l}\text { Class } \\
\text { (7) } \\
\end{array}$ & (8) & $\begin{array}{c}\log \left(L_{x}\right) \\
(9)\end{array}$ & $\begin{array}{c}\log \left(N_{H}\right) \\
(10)\end{array}$ & $\begin{array}{c}f_{3.6} \\
(\mu \mathrm{Jy}) \\
(11)\end{array}$ & $\begin{array}{c}f_{4.5} \\
(\mu \mathrm{Jy}) \\
(12)\end{array}$ & $\begin{array}{c}f_{5.8} \\
(\mu \mathrm{Jy}) \\
(13)\end{array}$ & $\begin{array}{c}f_{8.0} \\
(\mu \mathrm{Jy}) \\
(14)\end{array}$ & $\begin{array}{c}f_{24} \\
(\mu \mathrm{Jy}) \\
(15)\end{array}$ \\
\hline $84809.8+444901$ & -0.7 & 0.1 & 35.50 & -0.26 & 20.73 & unid & & & & $82.5 \pm 8.3$ & & $133.9 \pm 13.6$ & & \\
\hline J084812.4+445657 & 0 & 0.5 & 15.40 & $-c$ & 5 & unid & & & & & 210.2 & & & \\
\hline J084818.4+444844 & -0.9 & -0.0 & 8 & -0.40 & 20.66 & ELG & 0.405 & 42 & $<$ & $65.7 \pm 6.6$ & & 9 & & \\
\hline 884 & 1.8 & -1.5 & & -0.15 & 24.40 & unid & & & & & $<8$ & & -1 & \\
\hline $84824.6+445355$ & -0.8 & 0.3 & 2.61 & 0.03 & 22.21 & ELG & 0.747 & 42.7 & 22.4 & & & & & $711.8 \pm 10$ \\
\hline $084825.1+444808$ & -1.7 & -0.2 & 7.61 & -0.55 & 20.73 & BLAGN & 1.320 & 43.7 & $<$ & $84.5 \pm 8.5$ & & $104.5 \pm 10.6$ & & \\
\hline $084827.2+445433$ & -0.6 & -1.0 & 9.98 & -0.40 & 20.33 & BLAGN & 0.899 & 43.5 & $<$ & & & & & $1228.5 \pm 15$ \\
\hline J084827.4+445604 & 2.1 & -1.6 & 4.56 & 0.31 & 23.67 & ELG & 1.528 & 43.7 & 22.7 & & & & & $564.4 \pm 86$ \\
\hline $84830.2+445604$ & 0.0 & 0.0 & 2.55 & -0.18 & $>24.40$ & cont & & & & & & & & $<65.9$ \\
\hline $84831.6+445442$ & -0.3 & 0.7 & 2.44 & -0.15 & 25.42 & ELG & 1.2 & 43 & 22.0 & $11.8 \pm 1.2$ & $11.5 \pm 1.2$ & $10.6 \pm 1.6$ & $8.5 \pm 1.3$ & $<90.6$ \\
\hline
\end{tabular}

Note. Columns 4-10 present X-ray and optical source information from previous SEXSI publications (Harrison et al. 2003; Eckart et al. 2005; Eckart et al. 2006). (This table is available in its entirety in a machine-readable form in the online journal. A portion is shown here for guidance regarding its form and content.)

platescale of 2".45 pixel $^{-1}$. We convert the mosaics from surface brightness units to flux density units, and extract flux densities with SExtractor using an $11^{\prime \prime} .76$ diameter extraction aperture with an aperture correction of 1.697. Calibration with MIPS is performed with stars using a blackbody SED. Fadda et al. (2006) found that the color corrections for both starburst galaxies and AGNs were small at $z<1$. At $z>1$, the color correction for a prototypical luminous AGN (NGC 1068) remains small. As most SEXSI sources fall within one of these categories, we did not apply a color correction to account for MIPS sources having non-stellar SEDs. As with the IRAC photometry, we derive total uncertainties by adding in quadrature the statistical uncertainties (derived from SExtractor) to a conservative 10\% estimate of the systematic uncertainties (see, e.g., Engelbracht et al. 2007); for the MIPS data the statistical uncertainties are generally the dominant source of error. Compared with the IRAC photometry, a larger number of MIPS sources are detected with $\mathrm{S} / \mathrm{N}$ below $5(3<\mathrm{S} / \mathrm{N}<5)$. We flag these sources in the catalog and exclude them from our analysis. For the true non-detections we calculate $5 \sigma$ upper limits.

\subsection{Source Catalog}

Table 2 presents mid-infrared photometry for 290 hard X-ray-selected SEXSI sources. The full machine-readable catalog is available in the online journal. Each of the four IRAC catalogs as well as the MIPS catalog was individually matched to the SEXSI X-ray source positions ${ }^{8}$ using a 2".5 search radius. To calculate a false match rate we shifted the X-ray source catalog by $1^{\prime}$ and matched to the IRAC and MIPS catalogs; this entire procedure was repeated 6 times using different $1^{\prime}$ shifts. The resulting false match rates are $10.1 \%(3.6 \mu \mathrm{m}), 7.2 \%(4.5 \mu \mathrm{m})$, $3.7 \%(5.8 \mu \mathrm{m}), 2.6 \%(8.0 \mu \mathrm{m}), 1 \%(24 \mu \mathrm{m})$, and $<1 \%$ for fourband-detected IRAC sources. For ease of reference, Columns 4 10 of Table 2 present X-ray and optical source information from previous SEXSI publications, while the Spitzer mid-infrared data are presented in Columns 11-15. Complete X-ray source

\footnotetext{
8 We use the X-ray positions from Eckart et al. (2005) which have been corrected for small Chandra pointing offsets.
}

information is provided in Harrison et al. (2003) and opticalfollowup source catalogs are presented in Eckart et al. (2005, 2006).

Column 1 presents the source ID, followed by $\Delta \alpha$ and $\Delta \delta$, the difference in right ascension and declination between the $\mathrm{X}$-ray position and the Spitzer counterpart. Column 4 lists the 2-10 keV flux in units of $10^{-15} \mathrm{erg} \mathrm{cm}^{-2} \mathrm{~s}^{-1}$, while Column 5 gives the X-ray hardness ratio, $\mathrm{HR} \equiv(H-S) /(H+S)$, where $H$ is the hard-band counts $(2-10 \mathrm{keV})$ and $S$ is the soft-band counts $(0.5-2 \mathrm{keV})$. The optical-counterpart $R$-band magnitude is presented in Column 6, followed by the optical-spectroscopic classification (7) and the source redshift (8). Sources marked "unid" have no spectroscopic classification or redshift. The observed X-ray luminosity is given in Column 9 and the $N_{H}$ value determined from the X-ray data is found in Column 10. When the best-fit $N_{H}$ measurement is zero, we report " $<$ " in the table; see Eckart et al. (2006) for details of these calculations. Columns 11-14 provide the flux density in each of the IRAC bands with associated errors. The errors are $\gtrsim 10 \%$ of the source flux owing to our conservative estimate of systematic uncertainties for the Spitzer data. The MIPS data are presented in the final column. Entries in Columns 11-15 that are blank indicate that a source was not observed in that band.

\section{MID-INFRARED PROPERTIES OF X-RAY SELECTED AGNs}

This section explores the mid-infrared properties of the sample of 2-10 keV SEXSI sources. In particular, we focus on whether the X-ray-selected AGNs will also be identified as AGNs via the Stern et al. (2005) IRAC color-color AGN selection technique, and how this mid-infrared identification depends on various source properties, such as optical spectroscopic classification, redshift, X-ray flux, and mid-infrared flux density (Section 3.1), X-ray luminosity (Section 3.2), and $R$-band magnitude (Section 3.3). Section 3.5 provides a comparison to AGN samples presented in the literature: Section 3.5.1 compares the mid-infrared properties of hard X-ray-selected samples, while Section 3.5.2 discusses the identification of high-redshift, obscured quasars via 3.6-24 $\mu \mathrm{m}$ selection criteria. 


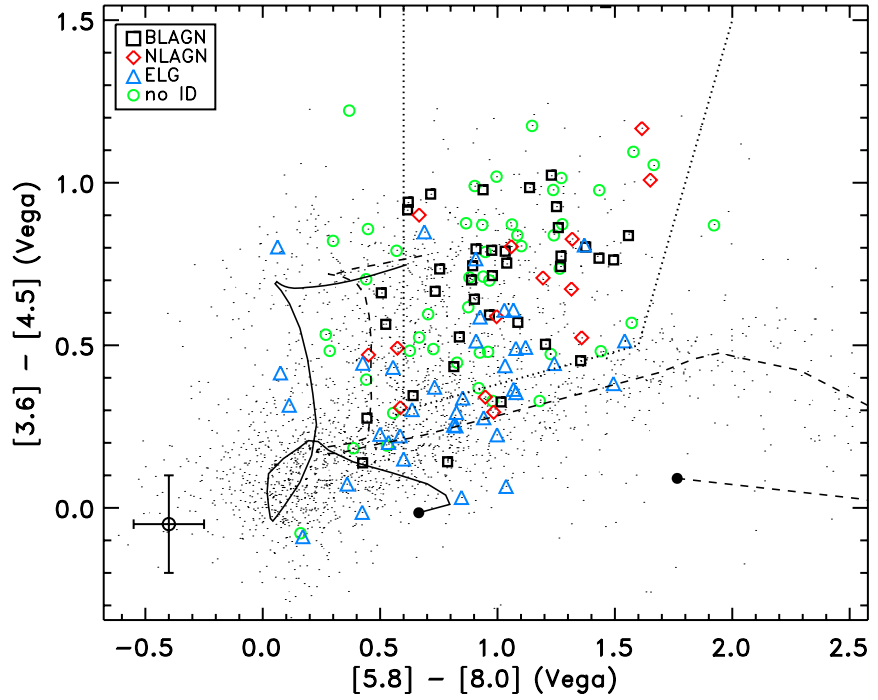

Figure 1. Color-color diagram of sources detected at $\geqslant 5 \sigma$ in all four IRAC bands (dots). Sources with a $2-10 \mathrm{keV}$ X-ray detection have a larger symbol overlaid, coded based on the optical spectroscopic classification (see inset). The dotted lines demarcate the AGN selection wedge introduced by Stern et al. (2005). The $0 \leqslant z \leqslant 3$ color tracks for two non-evolving galaxy templates from Devriendt et al. (1999) are illustrated; the large filled circles indicate $z=0$. A starburst galaxy is illustrated with the track of M82 (dashed line) and NGC 4429, an S0/Sa galaxy with a star formation rate $\sim 4000 \times$ lower, is indicated with the solid line. Median errors are illustrated at the lower left.

(A color version of this figure is available in the online journal.)

The Spitzer data cover 290 hard X-ray SEXSI sources, of which 154 have spectroscopic redshifts. However, a significant fraction of the X-ray sources does not have coverage in all four IRAC bands. ${ }^{9}$ For the following analysis we focus on the 189 hard X-ray sources $(65 \%)$ with observations in all four IRAC bands. For some analyses, we consider the slightly smaller sample of 155 sources $(53 \%)$ with MIPS $24 \mu \mathrm{m}$ observations as well. Because of the relative depths of the mid-infrared and X-ray data, nearly all of the SEXSI sources have infrared counterparts in one or more of the bands. Overall, $98 \%$ of SEXSI sources observed at $3.6 \mu \mathrm{m}$ are detected at greater than $5 \sigma$ at that wavelength, and the detection rates for the other IRAC bands are all $\gtrsim 90 \%$. Of the four-band IRAC-observed SEXSI sources, $57 \%$ have optical spectroscopic redshifts, and, of those, $89 \%$ are detected in all four IRAC bands. The corresponding MIPS detection rate is $54 \%$ (68\% if $<5 \sigma$ detections are included).

Figure 1 shows an IRAC color-color diagram of all midinfrared sources with $\geqslant 5 \sigma$ detections in all four IRAC bands (small black dots). Larger symbols indicate SEXSI 2-10 keV sources, as detailed in the caption. The dotted lines indicate the Stern et al. (2005) AGN selection wedge. We adopt the Stern et al. (2005) selection, which has slightly less contamination than the criteria suggested by Lacy et al. (2004) based on [4.5]-[8.0] versus [3.6]-[5.8] color space (e.g., Donley et al. 2008). The majority $(63 \%$, or $90 / 142)$ of the X-ray-selected AGNs lie in the Stern et al. (2005) selection wedge. Considering the spectroscopic sample, the AGN wedge contains $85 \%$ $(34 / 40)$ of the BLAGN, $64 \%(9 / 14)$ of NLAGN, and $34 \%$ $(13 / 38)$ of the ELG, as shown in Figure 1. Since there were only two ALGs (and one Galactic star) in the SEXSI sample

9 IRAC has two adjacent fields of view that are imaged in pairs (3.6 and $5.8 \mu \mathrm{m} ; 4.5$ and $8.0 \mu \mathrm{m})$. The sources in our sample that lack 4-band coverage typically lie nearer to the edge of the X-ray survey area and were imaged by only one IRAC FOV, thus yielding only 2-band coverage. with 4-band IRAC observations, these minor source populations are not considered further. Including $1 \sigma$ errors on the IRAC flux densities, all but three of the BLAGNs are consistent with the wedge (one of these is the lowest luminosity BLAGN in the sample, with $L_{2-10 \mathrm{keV}}=4 \times 10^{42} \mathrm{erg} \mathrm{s}^{-1}$ ) and all but one of the NLAGNs are consistent with the wedge selection. In the following sections, we explore the AGN selection further; in particular, we discuss the discrepancy between the high fraction of BLAGNs and NLAGNs selected using the mid-infrared techniques as compared to low fraction of ELGs. ${ }^{10}$

\subsection{Dependence on Observed X-ray Flux and Mid-infrared Flux Density}

Mid-infrared selection of AGNs relies exclusively on observed mid-infrared colors to distinguish AGNs from galaxies. This selection can be compared to X-ray identification of AGNs for various flux limits in both wavelength regimes. Figure 2 explores the wedge membership of our sample for flux cuts in the X-ray and mid-infrared. The top panels show that X-raybright AGNs are predominantly found in the wedge, while for fainter X-ray sources the wedge membership fraction decreases. However, the opposite is true when considering the mid-infrared data: a significant fraction of brighter X-ray-selected AGNs are found outside the wedge. Roughly half of the SEXSI AGNs brighter than $f_{3.6 \mu \mathrm{m}}=50 \mu \mathrm{Jy}([3.6]<16.86)$ are outside the wedge, compared to one-third for fainter mid-infrared sources. The non-wedge X-ray AGNs are also generally at low redshift.

Figure 2 (middle panels) also shows X-ray undetected midinfrared sources. At bright mid-infrared fluxes, most of the IRAC sources have colors consistent with low-redshift galaxies (e.g., see galaxy tracks in Figure 1). The X-ray sources outside the wedge have similar colors, suggesting that their mid-infrared flux is dominated by stellar emission as opposed to AGN activity. Many of these non-wedge-selected X-ray sources indeed have optical spectra of ELGs, consistent with the idea that emission related to star formation outshines the emission from the active nucleus in some parts of the spectrum (i.e., in the mid-infrared and optical); however, the X-ray luminosities of these sources are too high to be attributed wholly to emission related to star formation and instead we attribute the X-ray flux to emission from the nuclei. Figure 2 also shows that while a large fraction of the brightest mid-infrared IRAC AGN candidates are detected in the SEXSI Chandra images, this is not the case at fainter mid-infrared magnitudes: there is a large population of midinfrared-selected AGN candidates which lack X-ray detections, even in our relatively sensitive $\left(\left\langle t_{\exp }\right\rangle \approx 100 \mathrm{ks}\right)$ Chandra data sets.

Figure 3 shows the mid-infrared and X-ray flux distributions of SEXSI sources, and gives an indication of the efficiency of mid-infrared AGN selection as a function of the imaging depth in both wavelength regimes. The six fields considered here cover a relatively small area of the sky, and thus there are few bright sources in the sample. However, despite these small numbers, our finding that a relatively small proportion of SEXSI AGNs with bright mid-infrared fluxes are selected by the mid-infrared wedge shows that shallow infrared surveys will not be sensitive to the same AGN population as X-ray surveys. We consider these results in light of upcoming shallow, full-sky survey missions

\footnotetext{
10 Ten percent of the spectroscopically identified X-ray-selected AGNs lie at redshifts near that of the target cluster of the Chandra observations. Throughout Section 3 we include these sources in our analysis; however, we note that our conclusions remain unchanged if this $10 \%$ of sources is eliminated from the sample.
} 


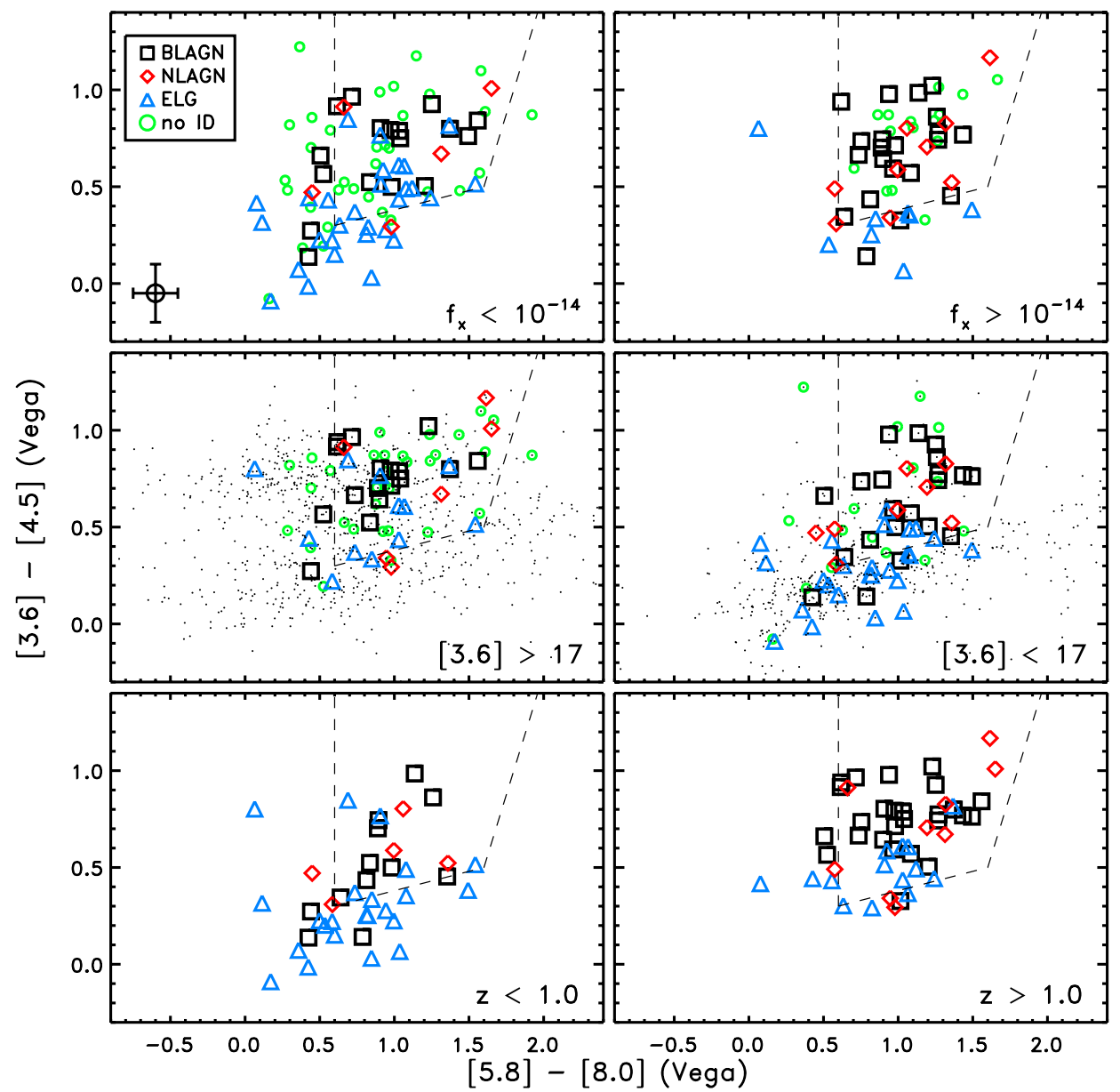

Figure 2. Mid-infrared wedge selection for samples divided by hard X-ray flux (upper panels; units of $\mathrm{erg} \mathrm{cm}^{-2} \mathrm{~s}^{-1}$ ), $3.6 \mu \mathrm{m}$ flux density (middle panels; Vega magnitudes) and redshift (lower panels). The upper left panel shows a median error bar for each color. Wedge selection is much more efficient at higher X-ray fluxes, as the brighter X-ray sources are predominantly BLAGNs. However, wedge selection is not more efficient at higher mid-infrared flux densities; a significant number of SEXSI ELGs outside of the wedge are brighter than [3.6] = $17(44 \mu \mathrm{Jy})$. The background dots in the middle panel show all 4-band IRAC sources in the SEXSI/Spitzer data; comparison to Figure 1 shows that the brighter sources have IRAC colors similar to starburst galaxies. The lower panel shows that the sources missed by the wedge are mainly at low redshift. Taken together, these results imply that shallow mid-infrared surveys would detect a large number of AGNs that have galaxy colors in the infrared, do not show strong AGN lines in their optical spectra, and are X-ray faint. These sources can only be readily identified with deep X-ray surveys.

(A color version of this figure is available in the online journal.)

such as the extended Röntgen Survey with an Imaging Telescope Array (eROSITA) and the Wide-field Infrared Survey Explorer (WISE) in our concluding section (Section 5.2).

\subsection{Dependence on X-ray and Infrared Luminosity}

Figure 4 illustrates the wedge selection for four 2-10 keV luminosity ranges, showing that X-ray luminosity strongly affects the fraction of sources which appear as AGNs in the mid-infrared. For this analysis, we use the rest-frame $2-10 \mathrm{keV}$ $\mathrm{X}$-ray luminosities from Eckart et al. (2006) that were derived assuming $\Gamma=1.5$. The entire lowest luminosity subsample $\left(L_{x}<10^{43} \mathrm{erg} \mathrm{s}^{-1}\right)$ lies either outside of the wedge or near the outskirts of the wedge, while the fraction within the wedge increases monotonically with X-ray luminosity. At the higher $\mathrm{X}$-ray luminosities $\left(L_{x}>10^{43.5} \mathrm{erg} \mathrm{s}^{-1}\right)$, all but two of the sources are consistent with the wedge. Of the 36 sources with spectroscopic identifications that are not selected by the wedge, 25 are classified as ELGs based on their optical spectra. These sources have a mean redshift of $\langle z\rangle \sim 0.8$, average infrared luminosity $\left\langle L_{4.5}\right\rangle \sim 10^{44} \mathrm{erg} \mathrm{s}^{-1}$, and average $\mathrm{X}$-ray luminosity $\left\langle L_{x}\right\rangle \sim 10^{43.2} \mathrm{erg} \mathrm{s}^{-1}$. Table 3 tabulates the fraction of X-ray sources in the wedge for the four plotted X-ray luminosity ranges. The X-ray luminosities used for binning the sources are the intrinsic, absorption-corrected luminosities described in Eckart et al. (2006); these results are not significantly different, however, if observed (e.g., uncorrected) luminosities are used instead.

Overall, it is clear that high X-ray luminosity sources predominantly show strong AGN features in their optical spectra and are wedge-selected, while the low-X-ray-luminosity sources generally lack AGN spectroscopic features and are not in the wedge. These results show that 2-10 keV X-ray surveys are efficient at finding low-luminosity AGNs, sources missed by mid-infrared selection techniques. This same trend is seen when the sample is divided by mid-infrared luminosity: sources with lower mid-infrared luminosities tend to fall out of the wedge. ${ }^{11}$ This is unsurprising given that, in luminous AGNs, nuclear emission reprocessed by dust dominates the mid-infrared continuum and

\footnotetext{
11 We do not apply $K$-corrections when calculating the mid-infrared luminosities as they are not well known. We estimate that for quasar-like spectra the $K$-correction will not be important, while for galaxy-like spectra it will vary a factor of $\approx 4-5$ between $z=0.5$ and $z=1.5$. Overall, these $K$-corrections will not change the fact that $L_{x}$ and $L_{\mathrm{IR}}$ are correlated, however, there will be scatter based on the particular SED.
} 


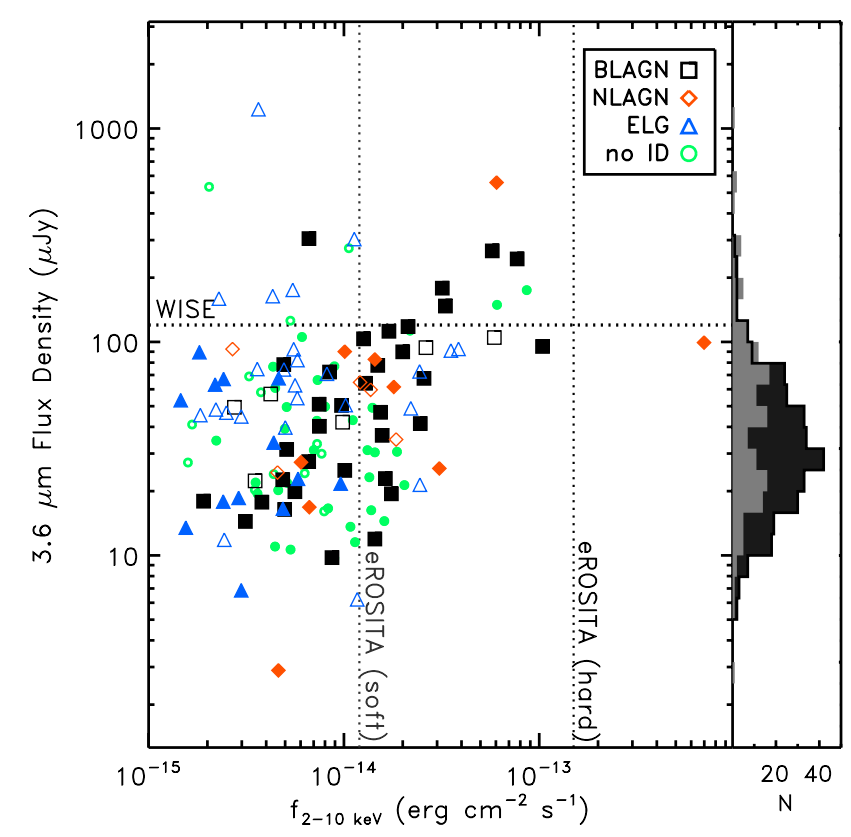

Figure 3. Mid-infrared flux density vs. hard X-ray flux for SEXSI sources. Filled symbols indicate sources within the Stern wedge. The histogram on the right shows the $3.6 \mu \mathrm{m}$ flux distribution for SEXSI AGNs (light gray) and all IRAC wedge sources from the SEXSI Spitzer fields (black). The dotted lines illustrate the depths that will be reached by two upcoming full-sky survey satellites: WISE (horizontal line) and eROSITA (vertical lines). The line labeled " $e$ ROSITA (hard)" provides the expected 2-10 keV sensitivity while the "eROSITA (soft)" line gives an indication of the $0.5-2 \mathrm{keV}$ sensitivity, scaled to the $2-10 \mathrm{keV}$-band using $\Gamma=1.9$. The ROSITA effective area is much larger below $2 \mathrm{keV}$, thus the mission will detect many more AGNs in the soft band. Some SEXSI AGNs brighter than the "eROSITA (soft)" limit but with spectra harder than $\Gamma=1.9$ will in fact not be detected by eROSITA.

(A color version of this figure is available in the online journal.)
Table 3

Wedge Selection as a Function of X-ray Luminosity

\begin{tabular}{lccccc}
\hline \hline \multirow{2}{*}{ Sample } & \multirow{2}{*}{$\log \left(L_{\min }\right)$} & $\log \left(L_{\max }\right)$ & \multicolumn{3}{c}{$L_{\min }<L_{x}<L_{\max }$} \\
\cline { 4 - 6 } & & & Wedge & Total & $\%$ \\
\hline All classes & \multirow{2}{*}{43.0} & 5 & 14 & $36 \pm 16$ \\
BLAGN & & & 1 & 2 & 50 \\
NLAGN & & & 0 & 1 & 0 \\
ELG & & & 4 & 11 & 36 \\
\hline All classes & 43.0 & 43.5 & 13 & 26 & $50 \pm 14$ \\
BLAGN & & & 4 & 5 & 80 \\
NLAGN & & & 2 & 2 & 100 \\
ELG & & & 7 & 19 & 37 \\
\hline All classes & 43.5 & 44.0 & 14 & 23 & $61 \pm 16$ \\
BLAGN & & & 11 & 14 & 79 \\
NLAGN & & & 1 & 2 & 50 \\
ELG & & & 2 & 7 & 29 \\
\hline All classes & 44.0 & $\cdots$ & 23 & 28 & $82 \pm 17$ \\
BLAGN & & & 17 & 18 & 94 \\
NLAGN & & & 6 & 9 & 67 \\
ELG & & & 0 & 1 & 0 \\
\hline
\end{tabular}

Note. The percentage errors represent the Poisson sampling uncertainties.

thus the mid-infrared luminosity is strongly tied to the AGN bolometric output.

\subsection{Dependence on R-band Magnitude}

Figure 5 shows this same color-color diagram as in Figures 1, 2 , and 4 but split at $R_{\text {cut }}=21$; the top panel shows Spitzer and SEXSI sources with bright optical counterparts and the bottom panel shows those with counterparts fainter than $R_{\text {cut }}=21$. Tabulated results comparing the wedge selection shown in these

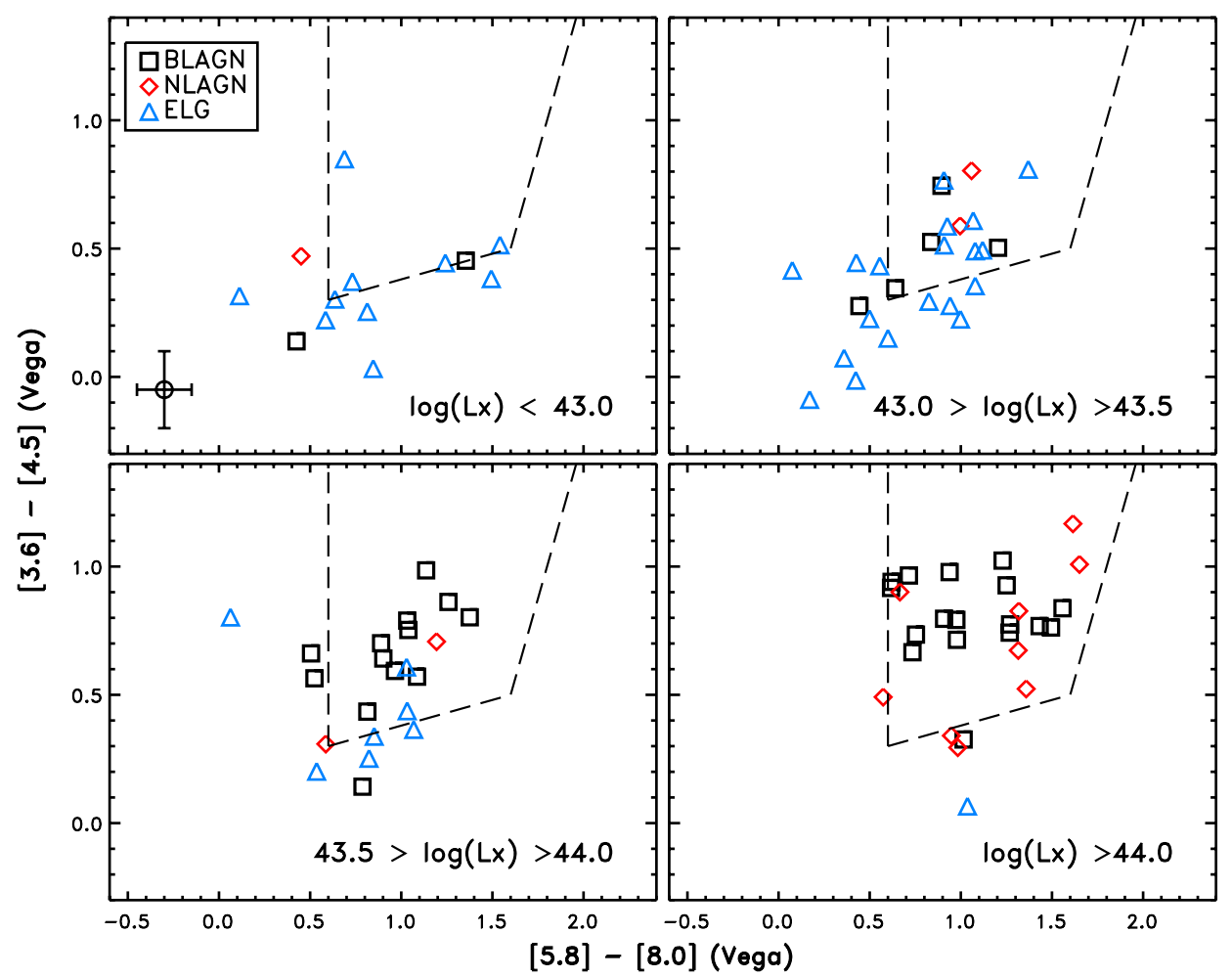

Figure 4. IRAC color-color diagram of SEXSI 2-10 keV sources, split by absorption-corrected intrinsic X-ray luminosity. As the X-ray luminosity grows, a higher fraction of sources fall solidly into the Stern et al. (2005) mid-infrared AGN selection wedge.

(A color version of this figure is available in the online journal.) 
Table 4

Wedge Selection as a Function of Optical Magnitude

\begin{tabular}{|c|c|c|c|c|c|c|c|c|c|c|c|c|}
\hline \multirow[t]{3}{*}{ Sample } & \multicolumn{6}{|c|}{ Bright: $R<21$} & \multicolumn{6}{|c|}{ Faint: $R>21$} \\
\hline & \multicolumn{3}{|c|}{ All 4-band IRAC } & \multicolumn{3}{|c|}{ SEXSI X-ray } & \multicolumn{3}{|c|}{ All 4-band IRAC } & \multicolumn{3}{|c|}{ SEXSI X-ray } \\
\hline & Wedge & Total & $\%$ & Wedge & Total & $\%$ & Wedge & Total & $\%$ & Wedge & Total & $\%$ \\
\hline All & 30 & 638 & $5 \pm 1$ & 19 & 27 & $70 \pm 16$ & 374 & 1298 & $29 \pm 2$ & 68 & 111 & $61 \pm 7$ \\
\hline BLAGN & & & & 13 & 13 & 100 & & & & 20 & 26 & 77 \\
\hline NLAGN & & & & 2 & 3 & 67 & & & & 7 & 11 & 64 \\
\hline ELG & & & & 1 & 6 & 17 & & & & 12 & 32 & 38 \\
\hline No ID & & & & 3 & 5 & 60 & & & & 29 & 42 & 69 \\
\hline
\end{tabular}

Note. The percentage errors represent the Poisson sampling uncertainties.
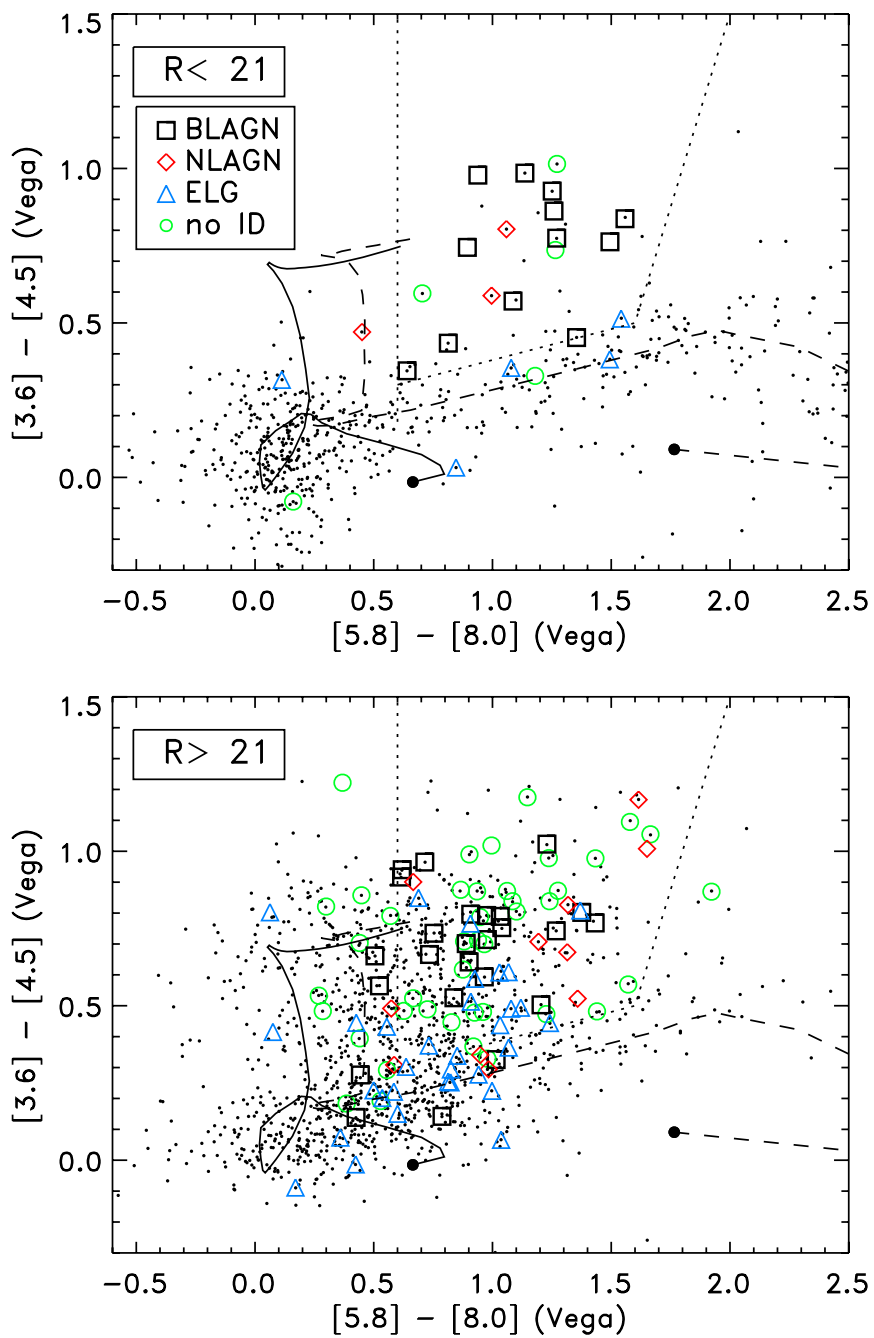

Figure 5. IRAC color-color diagram of sources with optical counterparts brighter (top panel) and fainter (bottom panel) than $R=21$. The galaxy tracks are the same as in Figure 1

(A color version of this figure is available in the online journal.)

plots are presented in Table 4. These data show a few strong trends. Only $5 \% \pm 1 \%$ of optically bright 4-band IRAC sources lie in the wedge, while $29 \% \pm 2 \%$ of the optically faint 4-band IRAC sources do. If we consider the subset of these sources that have $2-10 \mathrm{keV}$ detections, we see that the $70 \% \pm 16 \%$ of the optically bright $\mathrm{X}$-ray sources lie in the wedge and $61 \% \pm$ $7 \%$ of the optically faint X-ray sources are in the wedge. The $\mathrm{X}$-ray sources in general are more likely to fall in the wedge than a typical IRAC source, consistent with the notion that the wedge preferentially identifies AGNs (e.g., Gorjian et al.
2008; Ashby et al. 2009). An interesting trend evident from this sample is the large fraction of $R>21 \mathrm{X}$-ray undetected wedge sources: of the 374 IRAC sources in the wedge with $R>21$, only $18 \% \pm 2 \%$ (68 sources) are X-ray detected. For comparison, the corresponding optically bright $(R<21)$ sources are detected by Chandra nearly two-thirds $(63 \% \pm 14 \%)$ of the time. This trend is consistent with the idea that many of the X-ray undetected Spitzer AGNs lie at high redshift and/or are heavily obscured, resulting in faint optical counterparts and $\mathrm{X}$-ray non-detections. The infrared data are deep compared to the X-ray data, which, in combination with the scatter in the $\mathrm{X}$-ray to infrared flux ratios, means that sources that lie at high redshift may fall below the X-ray detection threshold yet have significant detections in the Spitzer data. Sources at moderate redshift with unobscured $2-10 \mathrm{keV}$ fluxes that would easily be detected in our Chandra data will fall below the detection threshold of even the deepest Chandra coverage in this study $\left(\sim 200 \mathrm{ks}\right.$, limiting flux of $\approx 2 \times 10^{-15} \mathrm{erg} \mathrm{cm}^{-2} \mathrm{~s}^{-1}$ ) if they are heavily obscured. For example, the observed flux of a source at $z=0.4$ with an unobscured $2-10 \mathrm{keV}$ flux of $f_{x} \approx 1 \times 10^{-14} \mathrm{erg} \mathrm{cm}^{-2} \mathrm{~s}^{-1}$ will be reduced to $f_{x}=$ $2 \times 10^{-15} \mathrm{erg} \mathrm{cm}^{-2} \mathrm{~s}^{-1}$ by an obscuring column of $1 \times 10^{24} \mathrm{~cm}^{-2}$, assuming photo-electric absorption and $\Gamma=1.9$. We also note that a significant number of the fainter wedge sources may also be normal galaxies (see also, e.g., Donley et al. 2008); the typical 600 s IRAC integrations considered here reach significantly $(\approx 3 \times)$ deeper than the shallow data sets from which the original wedge criteria were derived.

The final rows of Table 4 split the population by spectroscopic source type; the columns for Spitzer sources are blank here because we do not have spectroscopic followup of the nonX-ray-detected Spitzer AGN candidates, though follow-up of optically faint, mid-infrared-selected AGNs from the IRAC Shallow Survey shows a large fraction of them to be type 2 AGNs at $z \gtrsim 2$ (D. Stern et al. 2010, in preparation). Of the optically bright ELGs, only one of six sources lies within the wedge (and it is at the boundary), while 12 of $32 R>21$ ELGs lie in the wedge. This trend is different from that seen with the BLAGN and NLAGN, where a similar or higher fraction of $R<21$ sources lie in the wedge. This may indicate that for optically bright ELGs the starburst component of the SED is brighter than the AGN component pushing the source away from the AGN-defined wedge, while for some of the optically fainter sources the AGN component is strong in the mid-infrared leading to wedge selection. This trend is explained by higher $\mathrm{X}$-ray luminosity sources tending to fall in the wedge: as ELGs are identified at higher redshift they will be fainter in $R$ and also have higher average $L_{x}$ due to selection effects, and thus preferentially fall in the wedge as compared to low-luminosity, low-redshift sources. Splitting the wedge sources by redshift 
confirms this assertion (Figure 2). Overall, regardless of $R$ magnitude, the majority of the ELGs fall outside of the wedge.

\subsection{A Brief Summary of AGN Selection in the X-ray and Mid-infrared}

The results of our comparison of AGN selection techniques presented in this section may be summarized as follows.

1. Nearly two-thirds $(63 \%)$ of X-ray-selected AGNs lie in the mid-infrared selection wedge and nearly all of the X-rayselected AGNs that have optical spectra that exhibit highionization emission lines (i.e., BLAGNs and NLAGNs) lie within the mid-infrared selection wedge, within photometric uncertainties.

2. On average, the X-ray-selected AGNs classified as ELGs tend to fall outside of the infrared selection wedge, and thus are sources that would be missed by both mid-infrared and optical AGN selection techniques.

3. X-ray bright sources tend to fall in the AGN selection wedge, while sources with lower X-ray fluxes tend to fall outside the wedge.

4. The opposite is true when considering mid-infrared fluxes: the X-ray-selected AGNs that are faint in the mid-infrared tend to fall in the wedge, while those that are bright in the mid-infrared tend to be missed by the infrared AGN selection technique.

5. The non-wedge X-ray-selected AGNs generally lie at low redshift.

6. Overall, high X-ray luminosity sources fall within the midinfrared selection wedge, while the low-luminosity sources fall outside the wedge: the BLAGNs and NLAGNs are predominantly wedge selected, as they have higher X-ray luminosities, while the ELGs, which have lower X-ray luminosities, tend to fall outside the selection wedge.

7. There is a large population of mid-infrared, Spitzer-selected AGNs that is not detected in the $2-10 \mathrm{keV} X$-ray data.

8. When mid-infrared-selected AGN sources are split into optically bright $(R<21)$ and optically faint $(R>21)$ populations, the optically bright sources are detected by Chandra the majority of the time, while only $\approx 18 \%$ of the optically faint sources are identified in the X-ray.

\subsection{Comparison to Previous Work}

\subsubsection{Hard X-ray Selection}

We compare the SEXSI Spitzer sample to several other Chandra-Spitzer surveys that have a range of depth and area. The XMM-Newton Medium Deep Survey (XMDS; Pierre et al. 2007) identified 2-10 keV X-ray sources over $1 \mathrm{deg}^{2}$ with mid-infrared coverage from the Spitzer Wide-Area Infrared Extragalactic Survey survey (SWIRE; Lonsdale et al. 2003). Polletta et al. (2007) and Tajer et al. (2007) present a catalog of $122 \mathrm{X}$-ray-selected AGNs with $f_{x} \gtrsim 10^{-14} \mathrm{erg} \mathrm{cm}^{-2} \mathrm{~s}^{-1}$, about a factor of 5 shallower than the SEXSI sample considered here. All of these sources have at least 2-band IRAC detections. Using the Lacy et al. (2004) mid-infrared wedge selection technique, they find that $80 \%-95 \%$ of the X-ray sample would be selected on the basis of mid-infrared colors. We find that $82 \%$ of the XMDS AGNs are selected by the Stern wedge, much higher than the $62 \%$ of SEXSI sources within the wedge. If a flux cut of $f_{x}>10^{-14} \mathrm{erg} \mathrm{cm}^{-2} \mathrm{~s}^{-1}$ is applied to the SEXSI sources in order to match the limit of XMDS, the wedge membership increases to $74 \%$; when scatter due to photometric errors is considered, the wedge membership of the X-ray-brighter SEXSI sources is similar to XMDS (see upper panel of Figure 2).

Barmby et al. (2006) present Spitzer identifications for a catalog of $\sim 150$ X-ray AGNs in the Extended Groth Strip. The sample includes 138 sources detected in a $200 \mathrm{ks}$ Chandra exposure. Of these, 67 would be selected on the basis of hard-band detection and thus form a comparable sample to SEXSI. For the full catalog, Barmby et al. (2006) report that only $50 \%$ of sources are within the Stern wedge. Considering the hard-band-selected sample, this percentage increases to $57 \%$. If a further cut is made to select sources with $f_{x}>$ $2 \times 10^{-15} \mathrm{erg} \mathrm{cm}^{-2} \mathrm{~s}^{-1}$ (similar to the typical SEXSI limit), the percentage of wedge sources increases to $64 \%$, and for a flux cut of $f_{x}>1 \times 10^{-14} \mathrm{erg} \mathrm{cm}^{-2} \mathrm{~s}^{-1}$ (similar to XMDS) the percentage reaches $71 \%$. These trends show that various surveys agree when similar selection criteria are applied.

Our results are also consistent with those of Donley et al. (2007), who use deeper X-ray data over a smaller area from the Ms Chandra Deep Field-North (Alexander et al. 2003) and find that a small fraction of low- $L_{x}$ sources fit their mid-infrared power-law AGN selection criterion, with only one-third being within the Stern wedge. The mid-infrared selection efficiency for their sample increases from $\sim 14 \%$ at $L_{0.5-8 \mathrm{keV}}<10^{42} \mathrm{erg} \mathrm{s}^{-1}$ to $100 \%$ at $L_{0.5-8 \mathrm{keV}}>10^{44} \mathrm{erg} \mathrm{s}^{-1}$.

Combining these results, a consistent picture emerges in which hard X-ray surveys uncover a population of lowluminosity AGNs missed by mid-infrared selection techniques, with the efficiency of mid-infrared selection declining in proportion to the depth of the X-ray data. Optical selection techniques which rely on the detection of high-ionization narrow emission lines in order to identify obscured AGNs (e.g., Zakamska et al. 2003) similarly miss this population of low-luminosity AGNs.

Lastly, we can compare to the large sample of infraredselected AGNs assembled by Hickox et al. (2007) from the Boötes field. This sample includes $\sim 1500$ AGNs identified by the Stern wedge, with spectroscopic or photometric redshifts between $0.7<z<3$. The shallow X-ray coverage of this field results in few X-ray detections for the infrared-selected AGNs, but X-ray stacking shows that the infrared-selected candidates are, on average, consistent with AGN activity. These results show that while infrared and X-ray-selected samples do not identify exactly the same AGN populations, by and large they are both effective at constructing samples dominated by AGNs.

We note that broad consistency is found among these various works even though a variety of survey data and selection techniques were employed. In particular, the different applications of mid-infrared AGN color selection produce similar results, albeit with trade-offs in completeness and contamination. For comparison, we re-analyzed our sample using the Lacy et al. (2004) wedge selection technique, and we analyzed the Tajer et al. (2007) sample using the Stern et al. (2005) wedge. We find that the general trends and final conclusions are unchanged, though the numbers and percentages do vary. Studies, such as that recently undertaken by Assef et al. (2009), generally find that the Lacy et al. (2004) selection technique is more complete but is heavily contaminated by low-redshift star-forming galaxies. In comparison, the Stern et al. (2005) selection technique is less contaminated by low- and intermediate-redshift galaxies but suffers from some incompleteness, notably at $z \simeq 4.5$ as $\mathrm{H} \alpha$ shifts through the IRAC $3.6 \mu \mathrm{m}$ channel and for sources where the AGN emission is dominated by host galaxy stellar emission. The power-law AGN selection (Alonso-Herrero et al. 2006; Donley et al. 2007) is the least contaminated by non- 


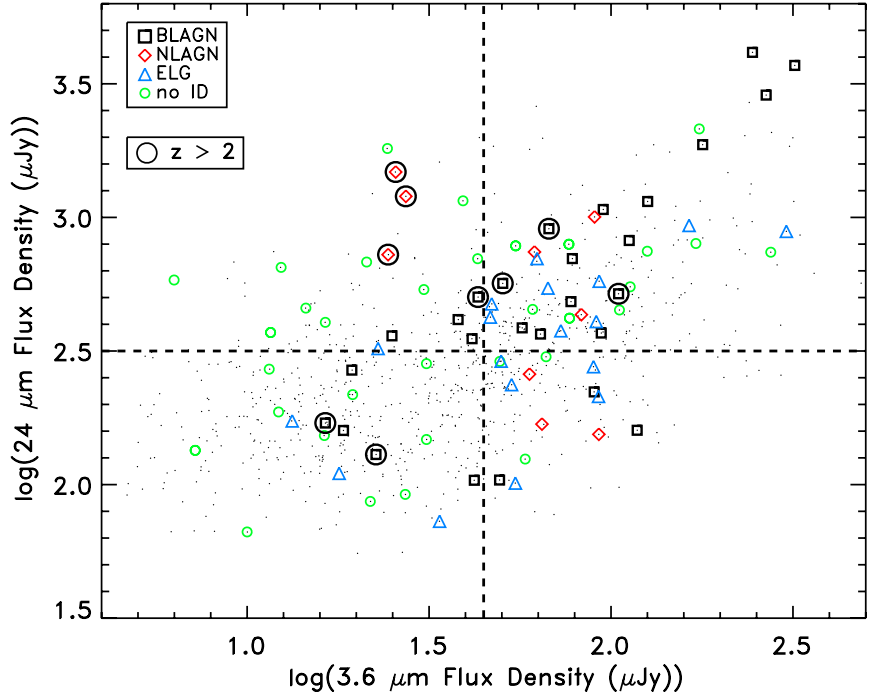

Figure 6. Upper left quadrant indicates the infrared selection area for highly obscured, $z>2$ type 2 AGNs, defined by Martínez-Sansigre et al. (2005). We plot sources with $\geqslant 5 \sigma$ detections at $3.6 \mu \mathrm{m}$ and $24 \mu \mathrm{m}$. Black circles surround all spectroscopically confirmed $z>2$ sources. This selection quadrant successfully identifies all three SEXSI-confirmed $z>2$ narrow-lined quasars. The ten spectroscopically unidentified SEXSI sources (no ID) in the selection quadrant are faint in the optical (typically $R \gtrsim 24$ ) and have X-ray properties that are consistent with the notion that they are indeed high-redshift, obscured quasars.

(A color version of this figure is available in the online journal.)

AGNs, but only selects a subset of the Stern et al. (2005) and Lacy et al. (2004) wedge-selected sources (see, e.g., Donley et al. 2008).

\subsubsection{High-redshift, Type-2 Quasar Selection}

Martínez-Sansigre et al. (2005) use the Spitzer FLS to search for type 2 quasars at $z>2$ using the following selection criteria: $f_{24 \mu \mathrm{m}} \geqslant 300 \mu \mathrm{Jy}, f_{3.6 \mu \mathrm{m}} \leqslant 45 \mu \mathrm{Jy}$, and $350 \mu \mathrm{Jy} \leqslant f_{1.4 \mathrm{GHz}} \leqslant$ $2 \mathrm{mJy}$. The $24 \mu \mathrm{m}$ cut is designed to define a reliable catalog of luminous quasar candidates, while the $3.6 \mu \mathrm{m}$ cut eliminates the unobscured quasars and the radio cut eliminates starburst galaxies. This effort identified 21 high-redshift, type 2 quasar candidates, ten of which were spectroscopically confirmed to be narrow-lined AGNs at $1.4<z<4$. The other 11 sources exhibit featureless optical spectra.

Figure 6 presents our sample of sources detected at both $24 \mu \mathrm{m}$ and $3.6 \mu \mathrm{m}$, with the upper-left quadrant containing the sources that meet the Martínez-Sansigre et al. (2005) infrared selection criteria. The large symbols indicate SEXSI $2-10 \mathrm{keV}$ sources, with confirmed $z>2$ sources circled in black. While our lack of deep radio data precludes application of the radio cut, SEXSI X-ray detections provide another effective method to avoid starburst contamination. Of the subsample of SEXSI sources with MIPS and IRAC coverage, we find that all three of the $z>2$ confirmed SEXSI NLAGNs fall in the selection quadrant, while the few confirmed broad-lined AGNs selected with these criteria all fall along the edges of the selection box. The high-redshift, narrow-lined sources exhibit the highionization UV emission lines indicative of an active nucleus, and have hard (2-10 keV) X-ray luminosities of $L_{x} \sim 10^{44.5} \mathrm{erg} \mathrm{s}^{-1}$. These sources exhibit hard X-ray spectra, with best-fit $N_{H}$ estimates of 1.4-9.4 $\times 10^{23} \mathrm{~cm}^{-2}$; two of the three sources do not have significant detections in the $0.5-2 \mathrm{keV}$ band. The sources at $z>2$ that exhibit broad AGN lines fall outside the quadrant (one sits just at the edge).
The majority (10 of 17) of the X-ray-selected sources that fall in the selection quadrant lack optical spectra. The SEXSI optical followup program focused on sources with $R \lesssim 23-24$, and thus many of the fainter $3.6 \mu \mathrm{m}$ sources, which tend to also be faint in $R$, lack spectroscopic coverage. However, the combined $\mathrm{X}$-ray and optical properties of the unidentified sources are consistent with high-luminosity, high-redshift, obscured AGNs. These ten sources are not detected in our $R$-band imaging or have magnitudes of $R>24$ and, if we assume $z=2$, have high $\mathrm{X}$-ray luminosities $\left(L_{2-10 \mathrm{keV}} \gtrsim 10^{44} \mathrm{erg} \mathrm{s}^{-1}\right)$. The sources are hard in the X-ray: many do not have significant detections in the $0.5-2 \mathrm{keV}$ band. Using the median hardness ratio of the sample and assuming $z=2$ and an intrinsic spectral index of $\Gamma=1.9$ we find $N_{H} \sim 3 \times 10^{23} \mathrm{~cm}^{-2}$. These properties are similar to those exhibited by the $z>2$ NLAGNs found by Martínez-Sansigre et al. (2005), as well as by the spectroscopically identified $z>2$ NLAGNs in our sample.

We thus confirm the result of Martínez-Sansigre et al. (2005) in a $2-10 \mathrm{keV} X$-ray-selected sample by showing that the 3.6-24 $\mu \mathrm{m}$ selection quadrant does indeed select high-redshift, highly obscured narrow-lined AGNs. Instead of using $1.4 \mathrm{GHz}$ radio selection criterion to eliminate starburst contamination we rely on a $2-10 \mathrm{keV}$ X-ray detection as an effective method to ensure that the sources are active galaxies. All of the spectroscopically confirmed $z>2$ narrow-lined AGNs in the SEXSI sample fall in the selection quadrant, and the SEXSI sources in the selection quadrant that lack spectroscopic information have X-ray hardness ratios and $R$-band characteristics consistent with the notion that they too are high-redshift, highly obscured quasars.

\section{X-RAY PROPERTIES OF MID-INFRARED-SELECTED AGNs}

A large number of sources in our mid-infrared sample have 4band IRAC detections but lack significant Chandra detections. In this section, we present results from an X-ray stacking analysis to study the mean X-ray properties of these sources, in particular focusing on the subset of these sources that fall within the mid-infrared AGN selection wedge. The aim is to first understand whether the mean X-ray properties of the wedge-selected sources differ from those outside the wedge, and whether these differences are consistent with the idea that the wedge-selected sources are active galaxies. A second goal is to estimate the intrinsic obscuration of the wedge-selected population using the hardness ratio of the stacked X-ray signals and assuming various population redshifts. We first describe the stacking methodology, and then present the results (Section 4.2) and a discussion (Section 4.3).

\subsection{X-ray Stacking Methodology}

The X-ray stacking analysis used tools provided by Chandra Interactive Analysis of Observations (CIAO) software and was aided greatly by acis_extract ${ }^{12}$ (ver. 3.91; Broos et al. 2002). We use CIAO (ver. 3.2) and CALDB (ver. 3.1) for the analysis presented here. Source lists for X-ray spectral extraction were assembled using our SExtractor catalogs of 4-band IRAC sources (with $>5 \sigma$ detections in each band) that fall within the Chandra images, excluding sources very near

\footnotetext{
12 Available at

http://www.astro.psu.edu/xray/docs/TARA/ae_users_guide.html.
} 
Table 5

Average Properties of X-ray Undetected IRAC Sources

\begin{tabular}{|c|c|c|c|c|c|c|c|c|c|c|}
\hline \multirow[t]{2}{*}{ Sample } & \multirow[t]{2}{*}{$N$} & \multicolumn{3}{|c|}{$0.5-8 \mathrm{keV}$} & \multicolumn{3}{|c|}{$0.5-2 \mathrm{keV}$} & \multicolumn{3}{|c|}{$2-8 \mathrm{keV}$} \\
\hline & & Cts & Sig. & $\begin{array}{c}f_{x} \\
\left(10^{-17}\right) \\
\end{array}$ & Cts & Sig. & $\begin{array}{c}f_{x} \\
\left(10^{-17}\right)\end{array}$ & Cts & Sig. & $\begin{array}{c}f_{x} \\
\left(10^{-17}\right)\end{array}$ \\
\hline All & 1298 & 699.2 & 18.7 & 10.4 & 427.3 & 19.8 & 2.1 & 271.9 & 8.9 & 9.2 \\
\hline Inside wedge & 226 & 243.5 & 14.5 & 19.1 & 123.8 & 12.8 & 3.3 & 119.6 & 8.7 & 21.4 \\
\hline Outside wedge & 1072 & 455.7 & 13.6 & 8.4 & 303.4 & 15.7 & 1.9 & 152.3 & 5.6 & 6.4 \\
\hline Inside extended wedge & 376 & 367.2 & 16.9 & 17.1 & 192.6 & 15.5 & 3.0 & 174.6 & 9.8 & 18.5 \\
\hline Outside extended wedge & 922 & 332.0 & 10.9 & 7.2 & 234.7 & 13.3 & 1.7 & 97.3 & 3.9 & 4.8 \\
\hline
\end{tabular}

Notes. $N$ indicates the number of sources in the particular sample, Cts provides the number of net backgroundsubtracted counts in each stacked sample, Sig. provides an estimate of the source significance $(S / \sqrt{B})$, and $f_{x}$ is the average X-ray flux for the given band in units of $10^{-17} \mathrm{erg} \mathrm{cm}^{-2} \mathrm{~s}^{-1}$. See Section 4.1

the edge of an ACIS chip. ${ }^{13}$ Sources that fall within $1 \mathrm{Mpc}$ of the center of each galaxy cluster target are eliminated from the stacking to reduce contamination from cluster galaxies.

Spectra were extracted for each source in the resulting catalog ( $\sim 2500$ sources). We choose $1.5 \mathrm{keV}$ as the energy at which the point-spread functions (PSFs) are computed and use extraction apertures that enclose $80 \%$ of the energy $(0.8 \times$ PSF). We calculated individual auxiliary response files (ARFs) and redistribution matrix files (RMFs) for each source, and we extracted a background spectrum from a local circular background region that includes at least 100 counts, taking care to mask out all detected X-ray sources (SEXSI sources, including soft-only X-ray sources, target point sources, and extended cluster emission). We scaled the background spectra based on the ratio of total exposure in the source extraction region to that of the background region.

Source counts and scaled background counts were tabulated for each source in several energy bands: the standard $0.5-8 \mathrm{keV}$ (full band), 2-8 keV (hard band), and $0.5-2 \mathrm{keV}$ (soft band), as well as $2-4 \mathrm{keV}, 4-6 \mathrm{keV}$, and 6-8 keV. Estimates of individual source significance were calculated for each band. Following Laird et al. (2005), we define detection significance as $S / \sqrt{B}$ and $\mathrm{S} / \mathrm{N}$ as $S / \sqrt{S+B}$, where $S$ and $B$ are the net source counts and scaled background counts, respectively. The average exposure per pixel (in each energy band) was calculated for each source by averaging the ARF over the given energy band and then over the source extraction cell. In addition to extracting $\mathrm{X}$-ray spectra at the position of the IRAC sources, as a control sample we repeated this procedure using a catalog with a $1^{\prime}$ offset applied to all source positions.

After calculating individual source statistics we stacked the sources. First, any IRAC sources that are within $5^{\prime \prime}$ of an $\mathrm{X}$-ray source were eliminated from the sample. In addition, we eliminated IRAC sources that lie within $5^{\prime \prime}$ of 64 sub-threshold $\mathrm{X}$-ray sources that were initially identified by wavdetect but later eliminated from the main SEXSI catalogs because they fall below the strict SEXSI $P<10^{-6}$ significance threshold. ${ }^{14}$ In field CL0848+44 we include only IRAC sources that fall on

\footnotetext{
13 These mid-infrared positions were then "uncorrected:" IRAC $3.6 \mu \mathrm{m}$ source positions were shifted to the uncorrected SEXSI X-ray frame by adding to each mid-infrared position the average X-ray-to-optical offset correction (see Section 3 and Table 2 of Eckart et al. 2005).

${ }^{14}$ In Harrison et al. (2003), we used wavdetect for initial source identification with the sigthresh parameter set to $10^{-5}$ to produce a complete catalog. In a subsequent step, we tested the significance of each source individually and eliminated sources from the source catalog with a nominal chance occurrence probability greater than $10^{-6}$. Sources that fall between the two thresholds were not included in the SEXSI X-ray sample.
}

ACIS chip 3, since the majority of the other X-ray sources are at very large off-axis angles.

The total signal in each band was calculated by summing the net background-subtracted counts from each source in the sample. To calculate an average flux we divided the summed signal by the sum of the average exposure per pixel in each

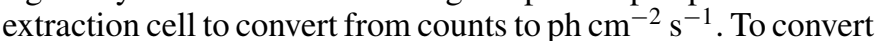
from $\mathrm{ph} \mathrm{cm}^{-2} \mathrm{~s}^{-1}$ to erg cm $\mathrm{cm}^{-2} \mathrm{~s}^{-1}$, we assumed a power-law spectrum with photon index $\Gamma=1.5$.

\subsection{Stacking Results}

We stacked three basic samples: all X-ray undetected sources (1298 sources), the wedge-selected sources (226 sources), and those that fall outside of the wedge (1072 sources). Table 5 presents a summary of the stacking results, where $N$ is the number of sources in the particular sample. The "Cts" column gives the total number of background-subtracted net counts in the sample. The "Sig" column gives an estimate of the significance of the stacked counts $\left(S_{\text {tot }} / \sqrt{B_{\text {tot }}}\right.$, see Section 4.1) and $f_{x}$ is the average X-ray flux for that band in units of $10^{-17} \mathrm{erg} \mathrm{cm}^{-2} \mathrm{~s}^{-1}$. The full sample of all X-ray undetected, 4band IRAC sources showed a significant detection in the stacked Chandra images. The stacks of the sources created by the shifted source catalog showed no significant detection in any band.

Stacking only the wedge-selected sources, we find significant detections in all three X-ray bands. The wedge-selected sample is, on average, brighter in the $\mathrm{X}$-ray: the average $0.5-8 \mathrm{keV}$ $\mathrm{X}$-ray flux from the wedge-selected IRAC sample is $\sim 2$ times larger than that of the full sample of 4-band IRAC sources. This trend is most significant in the hard band, where the average flux of the wedge-selected sample is over 3 times that of the sample that lies outside the wedge. These findings are consistent with the idea that AGNs preferentially lie inside the wedge.

To allow for errors in the IRAC photometry (e.g., see Figure 1), we also stacked based on an extended wedge defined by

$$
\begin{aligned}
([5.8]-[8.0])>0.5 & \wedge([3.6]-[4.5])>0.2 \cdot([5.8]-[8.0])+0.078 \\
& \wedge([3.6]-[4.5])>2.5 \cdot([5.8]-[8.0])-3.77,
\end{aligned}
$$

where $\wedge$ is the logical AND operator. This extended wedge is constructed by taking the original Stern et al. (2005) wedge and adding a swath of width $0.1 \mathrm{mag}$ at each edge. The stacking results for the samples inside and outside the extended wedge are also presented in Table 5; the extended wedge swath includes an additional 150 sources. Removing these 150 sources from the 1072 sources not selected by the original wedge removes a 
Table 6

Wedge-selected Stacked Spectrum: Implied $N_{H}$

\begin{tabular}{cc}
\hline \hline$z$ & $\begin{array}{c}N_{H} \\
\left(10^{22} \mathrm{~cm}^{-2}\right)\end{array}$ \\
\hline 0.0 & $1.3 \pm 0.2$ \\
0.5 & $3.5 \pm 0.6$ \\
1.0 & $7.4 \pm 1.4$ \\
1.5 & $14 \pm 3$ \\
2.0 & $24 \pm 5$ \\
3.0 & $48 \pm 9$ \\
\hline
\end{tabular}

third of the stacked counts in the hard band, leaving a source significance of only 3.9 in the hard-band sample of sources outside of the extended wedge. This exercise demonstrates that the hard X-ray emission, which is more easily produced by an AGN than by star formation, comes primarily from sources in or just outside the wedge, at least at our detection sensitivity.

Comparing the stacked fluxes of wedge-selected sources to those outside the wedge we find that the mid-infrared-selected AGNs are about 3 times brighter in the hard band than those outside the wedge, which is the same ratio we find if we calculate the average flux for SEXSI 2-10 keV-detected sources inside and outside the wedge. Although the wedge-selected sources are harder than those outside the wedge, the column density implied by the average hardness ratio is not extreme. Table 6 provides estimated $N_{H}$ values based on the hardness ratio of the stacked spectrum of the wedge-selected sample for assumed population redshifts ranging from 0.0 to 3.0. The $N_{H}$ values are calculated assuming an underlying power-law spectrum with $\Gamma=1.9$ and a Galactic column density of $2 \times 10^{20} \mathrm{~cm}^{-2}$; the associated uncertainties are estimated by propagating the Poisson sampling error in the hardness ratio. These $N_{H}$ estimates range from $\sim 1.3 \times 10^{22}$ to $4.8 \times 10^{23} \mathrm{~cm}^{-2}$, suggesting that the sources are intrinsically obscured, but are not, on average, Compton thick.

\subsection{Discussion of Stacking Results}

The hardness ratio of the wedge-selected stacked spectrum is consistent with moderate intrinsic obscuration, but is not suggestive of a highly obscured, Compton-thick source population. It is possible that the stacking procedure is missing a population of sources, such that the stacked counts are dominated by a subset of the less obscured wedge-selected sources. ${ }^{15} \mathrm{An}$ other possibility is that the assumption of an intrinsic $\Gamma=1.9$ power-law component plus photoelectric absorption is too simple, so that the estimated $N_{H}$ values are not representative of the sample. For example, it is possible that the sources within the sample have a range of power-law indices and/or absorbing columns. In addition, the soft X-ray emission may originate in a different location than the hard, power-law component, skewing the $N_{H}$ estimates to lower values. In reality, it is likely that some combination of these and other effects is operating. See also, e.g., Georgantopoulos et al. (2008).

Hickox et al. (2007) use an IRAC color-selection technique to identify a large sample of AGNs from the IRAC Shallow Survey, and an infrared/optical selection technique to separate the sources into unobscured (IRAGN1) and obscured (IRAGN2) sub-samples. They perform a similar stacking analysis of

\footnotetext{
15 We estimate that a lower limit of $\approx 30 \%$ of the sources must be contributing to the stacked signal: if it were fewer than $30 \%$ then individual source fluxes would fall above the Chandra detection threshold in order to reproduce the average stacked flux in the $2-8 \mathrm{keV}$ band. This provides an upper limit of $70 \%$ of the sources that are not contributing significantly to the stacked signal.
}

complementary X-ray data from the shallow (5 ks) Chandra XBoötes survey and find that the IRAGN1 are consistent with no obscuration and the IRAGN2 are consistent with $N_{H}$ $\sim 3 \times 10^{22} \mathrm{~cm}^{-2}$, independent of photometric redshift from $z_{\text {phot }} \approx 0.7-2.5$. The implied obscuring column density found using this shallow, wide-area data set is consistent with that of the SEXSI stacking analysis assuming $\left\langle z_{\text {stack }}\right\rangle \lesssim 1$. If the majority of the stacked emission in the SEXSI wedge-selected sample comes from a higher redshift population, then the implied $N_{H}$ is higher than that found in the XBoötes analysis. The average X-ray fluxes of X-ray undetected infrared AGNs from Hickox et al. (2007) are $f_{2-7 \mathrm{keV}} \sim 2 \times 10^{-15} \mathrm{erg} \mathrm{cm}^{-2} \mathrm{~s}^{-1}$, which is just at the hard-band SEXSI X-ray detection threshold, and more than an order of magnitude larger than the average stacked hard-band fluxes from the SEXSI analysis.

Several groups have explored the X-ray properties of small samples of optically identified type 2 quasars from the Sloan Digital Sky Survey using Chandra and XMM-Newton (e.g., Vignali et al. 2006; Ptak et al. 2006). These studies have found that approximately half of the type 2 quasars are detected in the $\approx 5-40$ ks X-ray images, and those that are detected exhibit moderate obscuring column densities $\left(\sim 10^{22}-10^{23} \mathrm{~cm}^{-2}\right)$. The sources that are undetected in the X-ray are likely highly obscured such that little $\mathrm{X}$-ray radiation below $10 \mathrm{keV}$ escapes. The high bolometric luminosities of these sources, as evidenced by both their mid-infrared properties and their optical spectra, imply highly obscured, high-luminosity AGNs that will require a sensitive high-energy ( $\gtrsim 10 \mathrm{keV}$ ) X-ray mission before they are detected in the X-rays.

\section{SUMMARY AND CONCLUSIONS}

We have presented an exploration of the relative efficiency of selecting AGNs in the X-ray with Chandra and in the mid-infrared with Spitzer. In this section, we summarize our comparison of the selection techniques and make predictions about the AGN samples that will be selected with future X-ray and mid-infrared surveys.

\subsection{Summary}

Two-thirds of the X-ray-selected AGNs are also selected by the Stern et al. (2005) Spitzer AGN selection criteria. Nearly all of the SEXSI X-ray sources that are classified as either broadline or narrow-line AGNs (i.e., those that exhibit high-ionization optical/UV emission lines in their optical spectra) are consistent with wedge selection within photometric uncertainties.

A large fraction of the low luminosity $\left(L_{2-10 \mathrm{keV}}<\right.$ $\left.10^{43.5} \mathrm{erg} \mathrm{s}^{-1}\right)$, hard X-ray (2-10 keV) AGNs identified by Chandra will be missed by Spitzer selection techniques. These low X-ray luminosity sources tend to lack the high-ionization emission lines that allow AGN identification via optical spectroscopy; thus, the sources that are missed by the Spitzer colorcolor AGN selection will also remain unidentified in optical spectroscopic surveys. These sources tend to lie at lower redshift and have higher fluxes in the infrared, on average. This suggests that many of these non-wedge-selected X-ray sources have mid-infrared fluxes that are dominated by stellar (starburst) emission as opposed to AGN activity; indeed, most have optical spectra of ELGs, consistent with the conclusion that emission related to star formation outshines the emission from the active nucleus in some parts of the spectrum (i.e., in the mid-infrared and optical), while the X-ray luminosities of these sources are dominated by X-ray emission from the active nuclei. In addition, 
we find that while a fair fraction $(\sim 63 \%)$ of the optically bright $(R<21)$ wedge-selected Spitzer AGNs are also identified by Chandra, over $80 \%$ of the optically faint wedge sources are not detected in the X-ray. It is likely that these fainter sources are AGNs that are heavily obscured and/or lie at high redshift. We also find that X-ray-selected high-redshift, type-2 quasars are also selected via the $3.6-24 \mu \mathrm{m}$ selection criteria proposed by Martínez-Sansigre et al. (2005); the radio selection criteria used by those authors to eliminate starburst contaminants are not necessary when the sources have X-ray properties indicative of AGN activity.

To explore the mean X-ray properties of the X-ray undetected, IRAC-selected AGN candidates, we have stacked the Chandra data extracted from the positions of IRAC sources. The stacked wedge sources show significant X-ray signals in the full, soft, and hard X-ray bands. The average hardness ratio of the stacked spectrum of sources inside the wedge is higher than that of sources outside of the wedge, and the average $2-8 \mathrm{keV}$ flux is 3 times larger. The hardness ratio of the wedge-selected stacked spectrum is consistent with moderate intrinsic obscuration $\left(N_{H}\right.$ $\sim 10^{22}-10^{23.7} \mathrm{~cm}^{-2}$ ), but is not suggestive of a highly obscured, Compton-thick source population.

\subsection{Conclusions}

The results of our study illustrate the challenges of identifying obscured and low-luminosity AGNs using any one technique. A complete understanding of AGN activity and black hole growth will require multiwavelength data sets: low-luminosity AGNs in galaxies with active star formation cannot be selected using mid-infrared color techniques, but are effectively found at low redshift with the current-generation of X-ray telescopes; obscured high-redshift objects are beyond the sensitivity threshold of Chandra and XMM, but they are effectively identified in the mid-infrared if the AGN luminosity is at the high end of the luminosity distribution.

The results from current X-ray and mid-infrared satellites enable us to estimate the progress possible with upcoming space missions that plan to conduct all-sky or pencil beam surveys. The WISE (Mainzer et al. 2005), which is scheduled for launch at the end of 2009, will provide full-sky mid-infrared imaging reaching a depth of $120 \mu \mathrm{Jy}$ at $3.3 \mu \mathrm{m}$ (similar to IRAC channel 1) and $160 \mu \mathrm{Jy}$ at $4.7 \mu \mathrm{m}$ (similar to IRAC channel 2). At these shallow depths, almost every source redder than [3.3]-[4.7] $\approx 0.5$ will be an AGN; high-redshift galaxies are too faint to make this flux cut, and the coolest brown dwarfs are too rare. The primary contaminant will be actively star-forming galaxies at redshifts of a few tenths, which should be readily identifiable from their optical morphologies and colors in relatively shallow imaging such as is available from the Sloan Digital Sky Survey. By applying a flux cut at the WISE depths to the Boötes IRAC survey (Eisenhardt et al. 2004; Ashby et al. 2009), we predict that WISE should detect approximately 85 AGN candidates per square degree, of which $\approx 15 \%$ will be low-redshift star-forming galaxy contaminants. WISE will therefore provide an unprecedented sample of high-luminosity obscured AGNs across a range of redshifts up to $z \sim 3$ identified based on mid-infrared color selection alone. WISE will also detect numerous other lowredshift $(z<1)$, low-luminosity $\left(L_{2-10 \mathrm{keV}} \lesssim 10^{43} \mathrm{erg} \mathrm{s}^{-1}\right)$ active galaxies, but the significant number of open symbols above the WISE threshold shown in Figure 3 shows that midinfrared colors alone are insufficient to identify them as AGNs.

The eROSITA (Predehl et al. 2006) X-ray mission, planned for launch in 2011, will provide full-sky hard X-ray images to

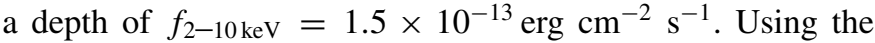
sample of XBoötes sources (Kenter et al. 2005) with fluxes above the ROSITA hard-band sensitivity limit, we predict that eROSITA should detect $\approx 4$ hard $\mathrm{X}$-ray sources per square degree. The mission will detect many more AGNs in the soft X-ray band because the effective area is much larger at energies below $2 \mathrm{keV}$, reaching soft-band depths of $f_{0.5-2 \mathrm{keV}}=$ $9 \times 10^{-15} \mathrm{erg} \mathrm{cm}^{-2} \mathrm{~s}^{-1}$. Scaling from the XBoötes $0.5-2 \mathrm{keV}$ sample we predict that $e$ ROSITA will detect $\approx 120$ sources per square degree in the soft band. Figure 3 shows that $e$ ROSITA will identify a large population of AGNs below the WISE threshold, most of which we project will be at $z \gtrsim 1$. The majority of these sources will be unobscured BLAGNs, but a significant population will be narrow-line, obscured AGNs (NLAGNs and ELG). However, eROSITA does not reach sufficient depth to detect many of the low-luminosity, low-redshift AGNs detected, but not identified as such, by WISE.

Clearly WISE and eROSITA combined will miss (or misidentify) low-luminosity obscured AGNs at all redshifts, most of which will also not be found in optical and soft X-ray surveys. At low redshift only a sensitive X-ray mission extending to $E_{x}>10 \mathrm{keV}$ will effectively uncover this population, which will be visible only in reprocessed light, if at all, at $E_{x}<10 \mathrm{keV}$. The Nuclear Spectroscopic Telescope Array (NuSTAR; Harrison et al. 2005), ${ }^{16}$ scheduled for launch in 2011, will make major advances in studying faint extragalactic sources at energies above $10 \mathrm{keV}$. NuSTAR will be the first focusing, high-energy $\mathrm{X}$-ray satellite and is designed to make targeted observations of the hard X-ray sky from 6-79 keV. NuSTAR, which has a FOV comparable to Chandra, plans to cover the Boötes and Great Observatories Origins Deep Survey (GOODS) fields, and should resolve $\gtrsim 50 \%$ of the $30 \mathrm{keV}$ X-ray background, reaching $10-40 \mathrm{keV}$ flux limits of $<10^{-14} \mathrm{erg} \mathrm{cm}^{-2} \mathrm{~s}^{-1}$. Many of the resolved sources are expected to be low-luminosity, nearby AGNs not identified as such in current mid-infrared or 2-10 keV X-ray surveys, and some NuSTAR sources may be AGNs with hard spectra owing to low-efficiency accretion. For studying the high-redshift, low-luminosity population, the International $X$-ray Observatory (IXO) will reach $2-10 \mathrm{keV}$ flux limits of $\sim 5 \times 10^{-17} \mathrm{erg} \mathrm{cm}^{-2} \mathrm{~s}^{-1}$ in a megasecond exposure with its wide-field imager. It could survey up to $10 \mathrm{deg}^{2}$ to a depth of $10^{-16} \mathrm{erg} \mathrm{cm}^{-2} \mathrm{~s}^{-1}$ and $0.25 \mathrm{deg}^{2}$ to a depth of $10^{-17} \mathrm{erg} \mathrm{cm}^{-2} \mathrm{~s}^{-1}$, uncovering the largest population of highredshift AGNs yet detected.

Predictions for the AGN populations that will be uncovered by these future missions must rely on careful studies of the AGNs identified by existing X-ray and infrared surveys. As illustrated in this paper, an understanding of the AGN selection techniques of the various missions, and their relative strengths and weaknesses, is crucial as data sets are synthesized to obtain an understanding of black hole growth and evolution in the universe.

Finally, we attempt to put the results of our study in the context of understanding the cosmic history of black hole growth and AGN accretion modes. The primary conclusions of this paper are that heavily obscured luminous AGNs are often missed by X-ray selection, while low-luminosity AGNs are often missed by mid-infrared selection. For the high-luminosity sources, various work has shown that, at least for the optically selected unobscured sources, most are emitting at close to their Eddington limit (e.g., Kollmeier et al. 2006). Multiwavelength observations are consistent with the heavily obscured high-

\footnotetext{
16 http://www.nustar.caltech.edu
} 
luminosity sources having similar intrinsic SEDs, simply with their UV, optical, and X-ray emission suffering from absorption.

As for the low-luminosity AGNs, there are two possibilities: they could be lower mass black holes again emitting at close to their Eddington limits, or they could be average-sized black holes, with masses similar to the high-luminosity sources, but simply emitting at lower Eddington ratios. Using a new set of empirical, low-resolution SED templates for AGNs and galaxies, Assef et al. (2009) shows that the likelihood of a source to be selected as an AGN using the Stern et al. (2005) mid-infrared color criteria is primarily a function of how strong the AGN is relative to the host galaxy-e.g., the Eddington ratio assuming that, to first order, the host galaxy mid-infrared emission scales with the galaxy total stellar mass which scales with the nuclear supermassive black hole mass. Thus, the fact that we find few low-luminosity sources using the mid-infrared criteria suggests that the low-luminosity sources are not emitting at close to their Eddington ratio. This is consistent with the results of Babić et al. (2007) who find that low-luminosity $\mathrm{X}$-ray sources in the Chandra Deep Field-South have a wide range of Eddington ratios, $10^{-5} \lesssim \log \left(L_{\text {bol }} / L_{\text {Edd }}\right) \lesssim 1$. One caveat on this interpretation is that it assumes that the AGN SED is independent of both Eddington ratio and luminositythe former of which has recently been questioned by Vasudevan \& Fabian (2007). Future work, in particular, higher energy observations with $N u S T A R$, will quantify the extent to which the intrinsic AGN SEDs depend on both luminosity and Eddington ratio.

This work is based on observations made with Spitzer, which is operated by the Jet Propulsion Laboratory, California Institute of the Technology under contract with the National Aeronautics and Space Administration (NASA). Support for this work was provided by NASA through award number 1314516 issued by JPL/Caltech. The authors thank the anonymous referee for his/her careful read and unusually diligent comments as well as Lewis Kotredes for assistance with Spitzer data reduction. M.E.E. acknowledges support from the NASA Postdoctoral Program.

\section{Facilities:: Spitzer, $C X O$, Keck:I, and Keck:II}

\section{REFERENCES}

Akiyama, M., Ueda, Y., Ohta, K., Takahashi, T., \& Yamada, T. 2003, ApJS, 148, 275

Akiyama, M., et al. 2000, ApJ, 532, 700

Alexander, D. M., et al. 2003, AJ, 126, 539

Alonso-Herrero, A., et al. 2006, ApJ, 640, 167

Antonucci, R. 1993, ARA\&A, 31, 473

Ashby, M. L. N., et al. 2009, ApJ, 701, 428

Assef, R. J., et al. 2009, ApJ, submitted (arXiv:0909.3849)

Babić, A., Miller, L., Jarvis, M. J., Turner, T. J., Alexander, D. M., \& Croom, S. M. 2007, A\&A, 474, 755

Barger, A. J., Cowie, L. L., Mushotzky, R. F., Yang, Y., Wang, W., Steffen, A. T., \& Capak, P. 2005, AJ, 129, 578

Barger, A., et al. 2003, AJ, 126, 632

Barmby, P., et al. 2006, ApJ, 642, 126

Bertin, E., \& Arnouts, S. 1996, A\&A, 117, 393

Brandt, W. N., \& Hasinger, G. 2005, ARA\&A, 43, 827

Broos, P., Townsley, L., Getman, K., \& Bauer, F. 2002, ACIS Extract, An ACIS Point Source Extraction Package (University Park, PA: Pennsylvania State Univ.)

Cattaneo, A., et al. 2009, Nature, 460, 213

Comastri, A., Setti, G., Zamorani, G., \& Hasinger, G. 1995, A\&A, 296, 1

Comastri, A., et al. 2002, ApJ, 571, 771

Devriendt, J. E. G., Guiderdoni, B., \& Sadat, R. 1999, A\&A, 350, 381

Donley, J. L., Rieke, G. H., Pérez-González, P. G., \& Barro, G. 2008, ApJ, 687, 111
Donley, J. L., Rieke, G. H., Pérez-González, P. G., Rigby, J. R., \& AlonsoHerrero, A. 2007, ApJ, 660, 167

Eckart, M. E., Laird, E. S., Stern, D., Mao, P. H., Helfand, D. J., \& Harrison, F. A. 2005, ApJS, 156, 35

Eckart, M. E., Stern, D., Helfand, D. J., Harrison, F. A., Mao, P. H., \& Yost, S. A. 2006, ApJS, 165, 19

Eisenhardt, P. R., et al. 2004, ApJS, 154, 48

Elitzur, M. 2008, New Astron. Rev., 52, 274

Engelbracht, C. W., et al. 2007, PASP, 119, 994

Fadda, D., et al. 2006, AJ, 131, 2859

Fazio, G. G., et al. 2004, ApJS, 154, 10

Ferrarese, L., \& Merritt, D. 2000, ApJ, 539, L9

Georgantopoulos, I., Georgakakis, A., Rowan-Robinson, M., \& Rovilos, E. 2008, A\&A, 484, 671

Gilli, R., Comastri, A., \& Hasinger, G. 2007, A\&A, 463, 79

Gorjian, V., et al. 2008, ApJ, 679, 1040

Harrison, F. A., Eckart, M. E., Mao, P. H., Helfand, D. J., \& Stern, D. 2003, ApJ, 596,944

Harrison, F. A., et al. 2005, Exp. Astron., 20, 131

Hasinger, G., Burg, R., Giacconi, R., Schmidt, M., Trumper, J., \& Zamorani, G. 1998, A\&A, 329, 482

Hasinger, G., Miyaji, T., \& Schmidt, M. 2005, A\&A, 441, 417

Heckman, T. M. 2008, arXiv:0809.1101

Hickox, R. C., et al. 2007, ApJ, 671, 1365

Hopkins, P. F., Hernquist, L., Cox, T. J., \& Kereš, D. 2008, ApJS, 175, 356

Hopkins, P. F., Richards, G. T., \& Hernquist, L. 2007, ApJ, 654, 731

Hora, J. L., et al. 2008, PASP, 120, 1233

John, T. L. 1988, A\&A, 193, 189

Kauffmann, G., \& Haehnelt, M. 2000, MNRAS, 311, 576

Kenter, A., et al. 2005, ApJS, 161, 9

Kollmeier, J. A., et al. 2006, ApJ, 648, 128

Krolik, J. H. 1999, Active Galactic Nuclei: From the Central Black Hole to the Galactic Environment (Princeton, NJ: Princeton Univ. Press)

La Franca, F., et al. 2002, ApJ, 570, 100

Lacy, M., et al. 2004, ApJS, 154, 166

Lacy, M., et al. 2005, ApJS, 161, 41

Laird, E. S., Nandra, K., Adelberger, K. L., Steidel, C. C., \& Reddy, N. A. 2005, MNRAS, 359, 47

Lonsdale, C. J., et al. 2003, PASP, 115, 897

Magorrian, J., et al. 1998, AJ, 115, 2285

Mainzer, A. K., Eisenhardt, P., Wright, E. L., Liu, F.-C., Irace, W., Heinrichsen, I., Cutri, R., \& Duval, V. 2005, Proc. SPIE, 5899, 262

Makovoz, D., \& Khan, I. 2005, in ASP Conf. Ser. 347, Astronomical Data Analysis Software and Systems XIV, ed. P. Shopbell, M. Britton, \& R. Ebert (San Francisco, CA: ASP), 81

Martínez-Sansigre, A., Rawlings, S., Lacy, M., Fadda, D., Marleau, F. R. Simpson, C., Willott, C. J., \& Jarvis, M. J. 2005, Nature, 436, 666

Nandra, K., \& Pounds, K. A. 1994, MNRAS, 268, 405

Pierre, M., et al. 2007, MNRAS, 382, 279

Polletta, M., et al. 2007, ApJ, 663, 81

Predehl, P., et al. 2006, Proc. SPIE, 6266, 19

Ptak, A., Zakamska, N. L., Strauss, M. A., Krolik, J. H., Heckman, T. M., Schneider, D. P., \& Brinkmann, J. 2006, ApJ, 637, 147

Richards, G. T., et al. 2006, AJ, 131, 2766

Rieke, G. H., et al. 2004, ApJS, 154, 25

Rosati, P., et al. 2002, ApJ, 566, 667

Scannapieco, E., Silk, J., \& Bouwens, R. 2005, ApJ, 635, L13

Schmidt, M., et al. 1998, A\&A, 329, 495

Seymour, N., et al. 2007, ApJS, 171, 353

Simpson, C., \& Eisenhardt, P. 1999, PASP, 111, 691

Steidel, C. C., Hunt, M. P., Shapley, A. E., Adelberger, K. L., Pettini, M., Dickinson, M., \& Giavalisco, M. 2002, ApJ, 576, 653

Stern, D., et al. 2005, ApJ, 631, 163

Szokoly, G. P., et al. 2004, ApJS, 155, 271

Tajer, M., et al. 2007, A\&A, 467, 73

Treister, E., et al. 2004, ApJ, 616, 123

Tremaine, S., et al. 2002, ApJ, 574, 740

Ueda, Y., Akiyama, M., Ohta, K., \& Miyaji, T. 2003, ApJ, 598, 886

Urry, C. M., \& Padovani, P. 1995, PASP, 107, 803

Vasudevan, R. V., \& Fabian, A. C. 2007, MNRAS, 381, 1235

Vignali, C., Alexander, D. M., \& Comastri, A. 2006, MNRAS, 373, 321

Weisskopf, M. C., O’dell, S. L., \& van Speybroeck, L. P. 1996, Proc. SPIE, 2805, 2

Werner, M. W., et al. 2004, ApJS, 154, 1

Wright, E. L., Eisenhardt, P., \& Fazio, G. 1994, in BAAS, 26, 893

Zakamska, N. L., et al. 2003, AJ, 126, 2125 